\title{
Kinetics of Radical Heterolysis Reactions Forming Alkene Radical Cations
}

\author{
John H. Horner*, Laurent Bagnol, Martin Newcomb*
}

\section{Supporting Information}

The synthetic routes to the radical precursors are shown in Schemes S1 and S2.

\section{Scheme S1}
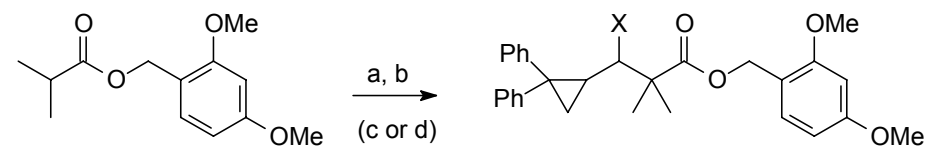

S1a: $X=\mathrm{OH}$

S1b: $X=O P(O)(O P h)_{2}$

S1c: $\mathrm{X}=\mathrm{OC}(\mathrm{O}) \mathrm{CF}_{3}$

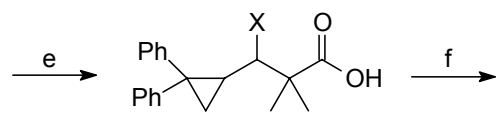

S2a: $X=O P(O)(O P h)_{2}$

S2b: $\mathrm{X}=\mathrm{OC}(\mathrm{O}) \mathrm{CF}_{3}$

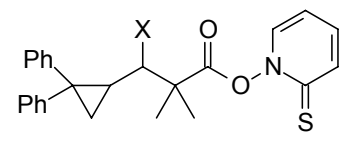

1d: $X=O P(O)(O P h)_{2}$

1e: $\mathrm{X}=\mathrm{OC}(\mathrm{O}) \mathrm{CF}_{3}$

${ }^{a}$ Reagents and conditions: (a) LDA, $-78^{\circ} \mathrm{C}$; (b) 2,2-diphenylcyclopropanecarboxaldehyde; (c) $(\mathrm{PhO})_{2}(\mathrm{O}) \mathrm{PCl}$; (d) $\mathrm{Et}_{3} \mathrm{~N},\left(\mathrm{CF}_{3} \mathrm{C}(\mathrm{O})\right)_{2} \mathrm{O}$; (e) $\mathrm{CAN}$; (f) 2,2'-dipyridyl disulfide bis- $N$-oxide, $\mathrm{Bu}_{3} \mathrm{P}$.

\section{Scheme S2}

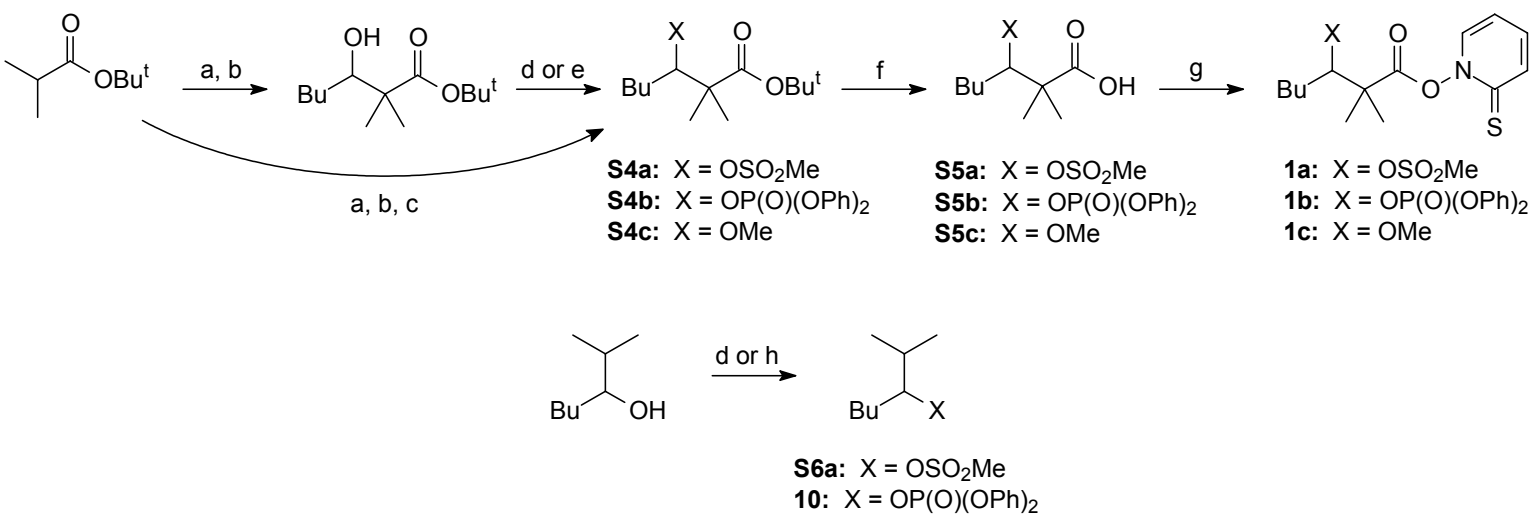

${ }^{a}$ Reagents and conditions: (a) LDA, $-78{ }^{\circ} \mathrm{C}$; (b) Pentanal; (c) $(\mathrm{PhO})_{2} \mathrm{P}(\mathrm{O}) \mathrm{Cl}$; (d) $\mathrm{Et}_{3} \mathrm{~N}, \mathrm{MeSO}_{2} \mathrm{Cl} / \mathrm{CH}_{2} \mathrm{Cl}_{2}$; (e) $\mathrm{NaH}$, $\mathrm{MeI} / \mathrm{THF}$; (f) $\mathrm{CF}_{3} \mathrm{COOH} / \mathrm{CH}_{2} \mathrm{Cl}_{2}$; (g) 2,2'-dipyridyl disulfide bis- $N$-oxide, $\mathrm{Bu}_{3} \mathrm{P}$; (h) $\mathrm{NaH}$, $(\mathrm{PhO})_{2} \mathrm{P}(\mathrm{O}) \mathrm{Cl} / \mathrm{THF}$.

General Information. Reactions involving water-sensitive reagents were performed under a nitrogen atmosphere. THF and diethyl ether were distilled from sodium-benzophenone ketyl. Alternatively THF, dichloromethane, and diethyl ether were saturated with argon and purified by passage through a bed of activated alumina under argon. ${ }^{\text {S1 }}$ Diisopropylamine and triethylamine were purified prior to use by distillation after stirring over calcium hydride 
overnight. Purification of reaction products was carried out by flash chromatography using Silicycle Ultra Pure Silica Gel (200-425 mesh) (60 A). Gas chromatography was performed on an Agilent Technologies 6890N gas chromatograph equipped with either a DB-5, $30 \mathrm{~m} \times 0.25$ $\mathrm{mm}$ (i.d.) column (using FID detection) or a DB-5, $15 \mathrm{~m} \mathrm{x} 0.53 \mathrm{~mm}$ (i.d.) (using TCD detection). Intermediate products typically were characterized by NMR spectroscopy and HRMS. NMR spectra were recorded in $\mathrm{CDCl}_{3}$ on a Varian Mercury-Vx-300 or Bruker AV-500 spetrometer at 300 or $500 \mathrm{MHz}\left({ }^{1} \mathrm{H}\right)$ and 75 or $125 \mathrm{MHz}\left({ }^{13} \mathrm{C}\right)$. Carbon type $\left(\mathrm{C}, \mathrm{CH}, \mathrm{CH}_{2}\right.$, or $\left.\mathrm{CH}_{3}\right)$ was assigned from DEPT spectra. The reported chemical shifts are downfield from tetramethylsilane $\left({ }^{1} \mathrm{H}\right.$ and ${ }^{13} \mathrm{C}$ ) or $\mathrm{H}_{3} \mathrm{PO}_{4}\left({ }^{31} \mathrm{P}\right)$. Mass spectra were obtained at the University of Illinois at Chicago Research Resources Center.

\title{
2,4-Dimethoxybenzyl-2,2-Dimethylpropanoate and 2,2-diphenylcyclopropane- carboxaldehyde were prepared according to the published procedures. ${ }^{\mathrm{S}}$
}

\begin{abstract}
2,4-Dimethoxybenzyl 3-hydroxy-3-(2,2-diphenylcyclopropyl)-2,2-dimethyl propanoate (S1a). To a stirred solution of diisopropylamine $(0.37 \mathrm{~mL}, 2.65 \mathrm{mmol})$ in $10 \mathrm{~mL}$ of THF was added $1.6 \mathrm{M} \mathrm{BuLi}$ in hexanes $(1.57 \mathrm{~mL}, 2.52 \mathrm{mmol})$ at $0{ }^{\circ} \mathrm{C}$. After stirring for $10 \mathrm{~min}$ at $0{ }^{\circ} \mathrm{C}$, the reaction mixture was cooled to $-78^{\circ} \mathrm{C}$, and a solution of 2,4-dimethoxybenzyl 2,2dimethylpropanoate $(0.5 \mathrm{~g}, 2.10 \mathrm{mmol})$ in $3 \mathrm{~mL}$ of THF was added dropwise. After stirring at $78^{\circ} \mathrm{C}$ for $30 \mathrm{~min}$, a solution of 2,2-diphenylcyclopropanecarboxaldehyde $(0.51 \mathrm{~g}, 2.30 \mathrm{mmol})$ in $5 \mathrm{~mL}$ of THF was added dropwise, and the reaction mixture was stirred and allowed to warm up to room temperature overnight. A saturated solution of $\mathrm{NH}_{4} \mathrm{Cl}$ and ether was added. The two phases were separated. The aqueous layer was extracted with $\mathrm{Et}_{2} \mathrm{O}$, and the combined organic layer was washed with brine and dried over $\mathrm{MgSO}_{4}$. Filtration and removal of the solvent under vacuum afforded $1.01 \mathrm{~g}$ of crude product. Flash chromatography (Hexanes/EtOAc: 4/1) afforded $0.58 \mathrm{~g}(53 \%)$ of a 5:1 mixture of diastereomers of the desired product as a viscous yellow oil. The major diastereomer was isolated, and carried on to the next step: ${ }^{1} \mathrm{H}-\mathrm{NMR}\left(\mathrm{CDCl}_{3}\right): \delta 7.30$ $7.11(\mathrm{~m}, 11 \mathrm{H}), 6.42-6.40(\mathrm{~m}, 2 \mathrm{H}), 5.10(\mathrm{AB}$ quartet, $J=12 \mathrm{~Hz}, \Delta v=18.6 \mathrm{~Hz}, 2 \mathrm{H}), 3.78(\mathrm{~s}, 3$ $\mathrm{H}), 3.71(\mathrm{~s}, 3 \mathrm{H}), 3.39-3.35(\mathrm{~m}, 1 \mathrm{H}), 2.11(\mathrm{~d}, J=7.5 \mathrm{~Hz}, 1 \mathrm{H}), 1.82-1.69(\mathrm{~m}, 2 \mathrm{H}), 1.20(\mathrm{~s}, 3 \mathrm{H})$, $1.18(\mathrm{~s}, 3 \mathrm{H}), 1.08(\mathrm{dd}, J=8.7,4.8 \mathrm{~Hz}, 1 \mathrm{H}) .{ }^{13} \mathrm{C} \mathrm{NMR}\left(\mathrm{CDCl}_{3}\right): \delta 177.05,161.22,158.84$, $146.70,141.00,131.21,129.78,128.31,128.25,126.34,125.93,116.55,103.86,98.56,73.23$, 62.32, 55.32, 48.13, 30.05, 27.98, 22.61, 21.30, 16.22. HRMS (ESI): $\mathrm{C}_{29} \mathrm{H}_{32} \mathrm{O}_{5}[\mathrm{M}+\mathrm{Na}]^{+}$calcd 483.2147; found 483.2140.
\end{abstract}

\section{2,4-Dimethoxybenzyl 3-(diphenylphosphatoxy)-3-(2,2-diphenylcyclopropyl)-2,2-}

dimethyl propanoate (S1b). To a stirred solution of diisopropylamine $(0.59 \mathrm{~mL}, 4.23 \mathrm{mmol})$ in $10 \mathrm{~mL}$ of THF was added $1.6 \mathrm{M} \mathrm{BuLi}$ in hexanes $(2.52 \mathrm{~mL}, 4.03 \mathrm{mmol})$ at $0{ }^{\circ} \mathrm{C}$. After stirring for $10 \mathrm{~min}$, the reaction mixture was cooled to $-78^{\circ} \mathrm{C}$, and a solution of 2,4 -dimethoxybenzyl 2,2-dimethylpropanoate $(0.8 \mathrm{~g}, 3.36 \mathrm{mmol})$ in $5 \mathrm{~mL}$ of THF was added dropwise. The reaction mixture was stirred at $-78^{\circ} \mathrm{C}$ for $30 \mathrm{~min}$. A solution of 2,2-diphenylcyclopropanecarboxaldehyde $(0.82 \mathrm{~g}, 3.70 \mathrm{mmol})$ in $5 \mathrm{~mL}$ of THF was added dropwise, and the reaction mixture was stirred at $-78{ }^{\circ} \mathrm{C}$ for $1.5 \mathrm{~h}$. Diphenyl chlorophosphate $(1.08 \mathrm{~g}, 4.03 \mathrm{mmol})$ was added, and the reaction mixture was stirred and allowed to warm up to room temperature overnight. A saturated solution of $\mathrm{NH}_{4} \mathrm{Cl}$ and ether was added. The reaction mixture was extracted with $\mathrm{Et}_{2} \mathrm{O}$, and the combined organic phase was washed with brine and dried over $\mathrm{MgSO}_{4}$. Filtration and removal of the solvent under vacuum afforded $2.53 \mathrm{~g}$ of crude product. Flash chromatography (hexanes/EtOAc: 4/1) afforded $1.56 \mathrm{~g} \mathrm{(67 \% )} \mathrm{of} \mathrm{a} \mathrm{6:1} \mathrm{mixture} \mathrm{of}$ diastereomers of the desired product as a viscous yellow oil. The major diastereomer was isolated and carried on in the next step. ${ }^{1} \mathrm{H}$ NMR $\left(\mathrm{CDCl}_{3}\right): \delta 7.32-6.99(\mathrm{~m}, 21 \mathrm{H}), 6.49-6.45(\mathrm{~m}$, $2 \mathrm{H}), 5.13(\mathrm{AB}$ quartet, $J=12.0 \mathrm{~Hz}, \Delta v=14.2 \mathrm{~Hz}, 2 \mathrm{H}), 4.58(\mathrm{t}, J=9.3 \mathrm{~Hz}, 1 \mathrm{H}), 3.79(\mathrm{~s}, 3 \mathrm{H})$, $3.75(\mathrm{~s}, 3 \mathrm{H}), 2.12(\mathrm{dt}, J=6.6,10.3 \mathrm{~Hz}, 1 \mathrm{H}), 1.55(\mathrm{t}, J=6.2 \mathrm{~Hz}, 1 \mathrm{H}), 1.35(\mathrm{~s}, 3 \mathrm{H}), 1.24(\mathrm{~s}, 3$ $\mathrm{H}), 1.21(\mathrm{dd}, J=10.2,5.1 \mathrm{~Hz}, 1 \mathrm{H}) .{ }^{13} \mathrm{C} \mathrm{NMR}\left(\mathrm{CDCl}_{3}\right): \delta 174.96,161.25,158.77,150.61\left(\mathrm{~d}, J_{\mathrm{C}-}\right.$ $\left.\mathrm{P}_{\mathrm{P}}=8.3 \mathrm{~Hz}\right), 150.50\left(\mathrm{~d}, J_{\mathrm{C}-\mathrm{P}}=7.3 \mathrm{~Hz}\right), 145.76,139.04,131.33,129.60,129.39,129.10,128.13$, $127.88,126.24,126.15,124.85,124.70,120.39\left(\mathrm{~d}, J_{\mathrm{C}-\mathrm{P}}=5.2 \mathrm{~Hz}\right), 119.78\left(\mathrm{~d}, J_{\mathrm{C}-\mathrm{P}}=5.2 \mathrm{~Hz}\right)$, 
$116.11,104.04,98.35,84.04\left(\mathrm{~d}, J_{\mathrm{C}-\mathrm{P}}=7.2 \mathrm{~Hz}\right), 61.99,55.25,55.19,48.27\left(\mathrm{~d}, J_{\mathrm{C}-\mathrm{P}}=4.1 \mathrm{~Hz}\right)$, $35.18,29.10\left(\mathrm{~d}, J_{\mathrm{C}-\mathrm{P}}=5.2 \mathrm{~Hz}\right), 24.25,18.41,16.59 .{ }^{31} \mathrm{P} \mathrm{NMR}\left(\mathrm{CDCl}_{3}\right): \delta-16.39$. HRMS (ESI): $\mathrm{C}_{41} \mathrm{H}_{41} \mathrm{O}_{8} \mathrm{P}[\mathrm{M}+\mathrm{K}]^{+}$calcd 731.2164; found 731.2160.

2,4-Dimethoxybenzyl 3-(trifluoroacetoxy)-3-(2,2-diphenylcyclopropyl)-2,2-dimethyl propanoate (S1c). Trifluoroacetic anhydride $(0.96 \mathrm{~mL}, 6.77 \mathrm{mmol})$ was added dropwise to a stirred solution of alcohol S1a $(2.6 \mathrm{~g}, 5.64 \mathrm{mmol})$ and triethylamine $(0.95 \mathrm{ml}, 6.77 \mathrm{mmol})$ in $20 \mathrm{~mL}$ of $\mathrm{CH}_{2} \mathrm{Cl}_{2}$ at $0{ }^{\circ} \mathrm{C}$. The reaction mixture was allowed to warm to room temperature over 3 h. Water was added, and the aqueous layer was extracted with $\mathrm{CH}_{2} \mathrm{Cl}_{2}$. The combined organic layer was washed with brine and dried over $\mathrm{MgSO}_{4}$. Filtration and removal of the solvent under vacuum afforded $3.75 \mathrm{~g}$ of crude material. Flash chromatography (Hexanes/EtOAc: 4/1) afforded $2.16 \mathrm{~g}(69 \%)$ of the desired product as a viscous yellow oil. ${ }^{1} \mathrm{H}$ NMR $\left(\mathrm{CDCl}_{3}\right): \delta 7.30$ $7.16(\mathrm{~m}, 11 \mathrm{H}), 6.47-6.44(\mathrm{~m}, 2 \mathrm{H}), 5.19(\mathrm{~d}, J=7.2 \mathrm{~Hz}, 1 \mathrm{H}), 5.10$ (AB quartet, $J=12 \mathrm{~Hz}, \Delta v=$ $14.6 \mathrm{~Hz}, 2 \mathrm{H}), 3.82(\mathrm{~s}, 3 \mathrm{H}), 3.74(\mathrm{~s}, 3 \mathrm{H}), 2.12(\mathrm{ddd}, J=9.9,7.5,7.2 \mathrm{~Hz}, 1 \mathrm{H}),(\mathrm{t}, J=5.6 \mathrm{~Hz}, 1$ $\mathrm{H}), 1.27(\mathrm{~s}, 3 \mathrm{H}), 1.19(\mathrm{dd}, J=9.0,4.8 \mathrm{~Hz}, 1 \mathrm{H}), 1.14(\mathrm{~s}, 3 \mathrm{H}) .{ }^{13} \mathrm{C} \mathrm{NMR}\left(\mathrm{CDCl}_{3}\right): \delta 174.62$, $161.40,159.03,145.94,139.70,131.25,129.62,128.90,128.65,128.44,126.90,126.63,116.51$, 104.06, 98.59, 80.45, 62.64, 55.50, 55.41, 47.72, 36.33, 25.56, 21.70, 21.57, 16.42. HRMS (ESI): $\mathrm{C}_{31} \mathrm{H}_{31} \mathrm{O}_{6} \mathrm{~F}_{3}[\mathrm{M}+\mathrm{Na}]^{+}$calcd 579.1970; found 579.1969.

3-(Diphenylphosphatoxy)-3-(2,2-diphenylcyclopropyl)-2,2-dimethylpropanoic acid (S2a). To a solution of ester $\mathbf{S 1 b}(0.75 \mathrm{~g}, 1.08 \mathrm{mmol})$ in 10:1 acetonitrile/water $(10 \mathrm{~mL})$, was added ceric ammonium nitrate $(1.3 \mathrm{~g}, 2.38 \mathrm{mmol})$. The reaction was stirred at room temperature for $1 \mathrm{~h}$ and quenched by addition of water. The resulting solution was extracted with $\mathrm{Et}_{2} \mathrm{O}$. The organic layers were combined and extracted with a $15 \%$ solution of $\mathrm{NaHSO}_{3}(5 \times 10 \mathrm{~mL})$. The organic layer was washed with brine and dried over $\mathrm{MgSO}_{4}$. Filtration and removal of the solvent under vacuum gave an orange oil. An NMR spectrum of the crude mixture showed quantitative conversion of the ester and formation of a mixture of the desired acid and 2,4dimethoxybenzaldehyde. The mixture was carried on in the next step without further purification. ${ }^{1} \mathrm{H} \mathrm{NMR}\left(\mathrm{CDCl}_{3}\right): \delta 8.21(\mathrm{br} \mathrm{s}, 1 \mathrm{H}), 7.26-6.95(\mathrm{~m}, 20 \mathrm{H}), 4.50(\mathrm{t}, J=9.3 \mathrm{~Hz}, 1 \mathrm{H})$, $2.04(\mathrm{dt}, J=3.3,9.6 \mathrm{~Hz}, 1 \mathrm{H}), 1.62(\mathrm{t}, J=6.0 \mathrm{~Hz}, 1 \mathrm{H}), 1.25(\mathrm{~s}, 3 \mathrm{H}), 1.23(\mathrm{~m}, 1 \mathrm{H}), 1.21(\mathrm{~s}, 3 \mathrm{H})$.

3-(Trifluoroacetoxy)-3-(2,2-diphenylcyclopropyl)-2,2-dimethylpropanoic acid (S2b). To a solution of ester S1c (2.16 g, $3.88 \mathrm{mmol})$ in 10:1 acetonitrile/water (40 mL), was added ceric ammonium nitrate $(4.68 \mathrm{~g}, 8.5 \mathrm{mmol})$. The reaction was stirred at room temperature for $1 \mathrm{~h}$ and quenched by addition of water. The resulting solution was extracted with $\mathrm{Et}_{2} \mathrm{O}$. The organic layers were combined and extracted with a $15 \%$ solution of $\mathrm{NaHSO}_{3}(5 \times 20 \mathrm{~mL})$. The organic pahse was washed with brine and dried over $\mathrm{MgSO}_{4}$. Filtration and removal of the solvent under vacuum gave an orange oil. An NMR spectrum of the crude mixture showed quantitative conversion of the ester and formation of a mixture of the desired acid and 2,4-dimethoxybenzaldehyde. The mixture was carried on in the next step without further purification. ${ }^{1} \mathrm{H}$ $\operatorname{NMR}\left(\mathrm{CDCl}_{3}\right): \delta 7.29-7.19(\mathrm{~m}, 10 \mathrm{H}), 5.11(\mathrm{~d}, J=8.1 \mathrm{~Hz}, 1 \mathrm{H}), 2.16(\mathrm{dt}, J=6.0,8.4 \mathrm{~Hz}, 1 \mathrm{H})$, $1.85(\mathrm{t}, J=5.6 \mathrm{~Hz}, 1 \mathrm{H}), 1.31(\mathrm{~s}, 3 \mathrm{H}), 1.24(\mathrm{dd}, J=8.7,4.5 \mathrm{~Hz}, 1 \mathrm{H}), 1.14(\mathrm{~s}, 3 \mathrm{H})$.

(1H)-2-Thioxo-1-pyridyl 3-(diphenylphosphatoxy)-3-(2,2-diphenylcyclopropyl)-2,2dimethylpropanoate (1d). To a solution of acid $\mathbf{S 2 a}(1.3 \mathrm{mmol})$ and 2,2'-dipyridyl disulfide bis- $N$-oxide $(0.361 \mathrm{~g}, 1.43 \mathrm{mmol})$ in $\mathrm{CH}_{2} \mathrm{Cl}_{2}(12 \mathrm{~mL})$ was added tributylphosphine $(0.36 \mathrm{~mL}$, $1.43 \mathrm{mmol}$ ) in the dark at $0{ }^{\circ} \mathrm{C}$. After $2 \mathrm{~h}$ of stirring in the dark at room temperature, aqueous $\mathrm{Na}_{2} \mathrm{CO}_{3}(10 \%)$ was added to the reaction mixture. The phases were separated, and the aqueous layer was extracted with $\mathrm{CH}_{2} \mathrm{Cl}_{2}$. The combined organic phase was washed with brine and dried over $\mathrm{MgSO}_{4}$. Filtration and removal of the solvent under vacuum afforded $1.08 \mathrm{~g}$ of crude

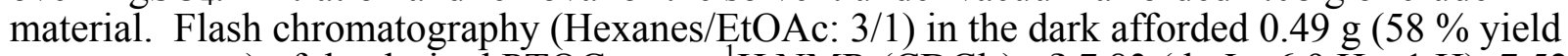
over two steps) of the desired PTOC ester. ${ }^{1} \mathrm{H}$ NMR $\left(\mathrm{CDCl}_{3}\right): \delta 7.83(\mathrm{~d}, J=6.9 \mathrm{~Hz}, 1 \mathrm{H}), 7.52$ $(\mathrm{dd}, J=8.7,1.5 \mathrm{~Hz}, 1 \mathrm{H}), 7.27-6.90(\mathrm{~m}, 21 \mathrm{H}), 6.23(\mathrm{dt}, J=1.8,6.9 \mathrm{~Hz}, 1 \mathrm{H}), 4.58(\mathrm{dd}, J=10.2$, $7.5 \mathrm{~Hz}, 1 \mathrm{H}), 2.10(\mathrm{dt}, J=6.6,9.6 \mathrm{~Hz}, 1 \mathrm{H}), 1.79(\mathrm{t}, J=6.0 \mathrm{~Hz}, 1 \mathrm{H}), 1.65(\mathrm{~s}, 3 \mathrm{H}), 1.58(\mathrm{~s}, 3 \mathrm{H})$, 
$1.42(\mathrm{dd}, J=9.3,6.0 \mathrm{~Hz}, 1 \mathrm{H}) .{ }^{13} \mathrm{C} \mathrm{NMR}\left(\mathrm{CDCl}_{3}\right): \delta 175.78(\mathrm{C}), 170.83(\mathrm{C}), 161.85(\mathrm{C}), 150.33$ (C), $145.44(\mathrm{C}), 139.17(\mathrm{CH}), 138.71(\mathrm{C}), 136.84(\mathrm{CH}), 133.55(\mathrm{CH}), 129.65(\mathrm{CH}), 129.49$ $(\mathrm{CH}), 128.36(\mathrm{CH}), 128.21(\mathrm{CH}), 126.63(\mathrm{CH})\left(\mathrm{d}, J_{\mathrm{C}-\mathrm{P}}=8.2 \mathrm{~Hz}\right), 125.35(\mathrm{CH}), 125.00(\mathrm{CH})$, $120.29(\mathrm{CH})\left(\mathrm{d}, J_{\mathrm{C}-\mathrm{P}}=4.1 \mathrm{~Hz}\right), 119.93(\mathrm{CH})\left(\mathrm{d}, J_{\mathrm{C}-\mathrm{P}}=5.2 \mathrm{~Hz}\right), 112.23(\mathrm{CH}), 84.84(\mathrm{CH})\left(\mathrm{d}, J_{\mathrm{C}-\mathrm{P}}\right.$ $=7.2 \mathrm{~Hz}), 49.19(\mathrm{C}), 34.50(\mathrm{C}), 27.30(\mathrm{CH})\left(\mathrm{d}, J_{\mathrm{C}-\mathrm{P}}=8.2 \mathrm{~Hz}\right), 24.55\left(\mathrm{CH}_{3}\right), 18.98\left(\mathrm{CH}_{3}\right), 18.16$ $\left(\mathrm{CH}_{2}\right) .{ }^{3} \mathrm{P}$ NMR $\left(\mathrm{CDCl}_{3}\right): \delta$-14.96. $\mathrm{HRMS}(\mathrm{ESI}): \mathrm{C}_{37} \mathrm{H}_{34} \mathrm{NSPO}_{6}[\mathrm{M}+\mathrm{Na}]^{+}$calcd 674.1742; found 674.1740 .

(1H)-2-Thioxo-1-pyridyl 3-(trifluoroacetoxy)-3-(2,2-diphenylcyclopropyl)-2,2dimethylpropanoate (1e). To a solution of acid $\mathbf{S 2 b}(3.88 \mathrm{mmol})$ and 2,2'-dipyridyl disulfide bis- $N$-oxide $(1.08 \mathrm{~g}, 4.27 \mathrm{mmol})$ in dry $\mathrm{CH}_{2} \mathrm{Cl}_{2}(35 \mathrm{~mL})$ was added tributylphosphine $(1.07 \mathrm{~mL}$, $4.27 \mathrm{mmol}$ ) in the dark at $0{ }^{\circ} \mathrm{C}$. After $2 \mathrm{~h}$ of stirring in the dark at room temperature, aqueous $\mathrm{Na}_{2} \mathrm{CO}_{3}(10 \%)$ was added to the reaction mixture. The phases were separated, and the aqueous layer was extracted with $\mathrm{CH}_{2} \mathrm{Cl}_{2}$. The combined organic layer was washed with brine and dried over $\mathrm{MgSO}_{4}$. Filtration and removal of the solvent under vacuum afforded $3.12 \mathrm{~g}$ of crude material. Flash chromatography (Hexanes/EtOAc: 3/1) in the dark afforded $0.82 \mathrm{~g}(41 \%$ yield over two steps) of the desired PTOC ester. ${ }^{1} \mathrm{H} \mathrm{NMR}\left(\mathrm{CDCl}_{3}\right): \delta 7.64(\mathrm{dd}, J=1.8,10.8 \mathrm{~Hz}, 1 \mathrm{H})$, 7.39-7.14 (m, $12 \mathrm{H}), 6.58(\mathrm{dt}, J=1.1,6.9 \mathrm{~Hz}, 1 \mathrm{H}), 5.14(\mathrm{~d}, J=7.5 \mathrm{~Hz}, 1 \mathrm{H}), 2.40(\mathrm{dt}, J=7.5,6$ $\mathrm{Hz}, 1 \mathrm{H}), 1.84(\mathrm{t}, J=5.6 \mathrm{~Hz}, 1 \mathrm{H}), 1.52(\mathrm{~s}, 3 \mathrm{H}), 1.46(\mathrm{~s}, 3 \mathrm{H}), 1.29(\mathrm{dd}, J=5.3,9.3 \mathrm{~Hz}, 1 \mathrm{H})$. ${ }^{13} \mathrm{C}$ NMR $\left(\mathrm{CDCl}_{3}\right): \delta 175.72(\mathrm{C}), 170.14(\mathrm{C}), 145.13(\mathrm{C}), 139.14(\mathrm{C}), 137.41(\mathrm{CH}), 137.15$ $(\mathrm{CH}), 133.48(\mathrm{CH}), 129.37(\mathrm{CH}), 128.62(\mathrm{CH}), 128.57(\mathrm{CH}), 128.46(\mathrm{CH}), 127.03(\mathrm{CH}), 126.67$ $(\mathrm{CH}), 112.74(\mathrm{CH}), 80.62(\mathrm{CH}), 47.53(\mathrm{C}), 36.53(\mathrm{C}), 24.96(\mathrm{CH}), 22.13\left(\mathrm{CH}_{3}\right), 21.11\left(\mathrm{CH}_{3}\right)$, $16.33\left(\mathrm{CH}_{2}\right)$. HRMS (ESI): $\mathrm{C}_{27} \mathrm{H}_{24} \mathrm{NSO}_{4} \mathrm{~F}_{3}[\mathrm{M}+\mathrm{Na}]^{+}$calcd 538.1276; found 538.1284.

1-(2,2-Diphenylcyclopropyl)-2-methylpropene. To a solution of hexamethyldisilazane $(0.56 \mathrm{~g}, 3.49 \mathrm{mmol})$ in $5 \mathrm{~mL}$ of THF was added $1.6 \mathrm{M} \mathrm{BuLi}$ in hexanes $(2.1 \mathrm{~mL}, 3.37 \mathrm{mmol})$ at 0 ${ }^{\circ} \mathrm{C}$. After stirring for $15 \mathrm{~min}$, isopropyltriphenylphosphonium iodide $(1.46 \mathrm{~g}, 3.37 \mathrm{mmol})$ was added, and the reaction mixture was stirred for $1 \mathrm{~h}$ at $0{ }^{\circ} \mathrm{C}$. A solution of 2,2diphenylcyclopropanecarboxaldehyde $(0.5 \mathrm{~g}, 2.25 \mathrm{mmol})$ in $5 \mathrm{~mL}$ of THF was added, and the reaction mixture was stirred and allowed to warm up overnight. After filtration, the solvent was removed under vacuum. Flash chromatography (Hexanes/EtOAc: 99/1) afforded $350 \mathrm{mg}(63 \%)$ of the desired alkene as a colorless liquid. ${ }^{1} \mathrm{H} \operatorname{NMR}\left(\mathrm{CDCl}_{3}\right): \delta 7.25-7.00(\mathrm{~m}, 10 \mathrm{H}), 4.28(\mathrm{~d}, J=$ $10.9 \mathrm{~Hz}, 1 \mathrm{H}), 2.23(\mathrm{dt}, J=6.0,9.2 \mathrm{~Hz}, 1 \mathrm{H}), 1.72(\mathrm{~d}, J=1.2 \mathrm{~Hz}, 3 \mathrm{H}), 1.52(\mathrm{~d}, J=0.9 \mathrm{~Hz}, 3$ $\mathrm{H}), 1.49(\mathrm{dd}, J=9.0,4.8 \mathrm{~Hz}, 1 \mathrm{H}), 1.28(\mathrm{dd}, J=6.0,4.8 \mathrm{~Hz}, 1 \mathrm{H}) .{ }^{13} \mathrm{C} \mathrm{NMR}\left(\mathrm{CDCl}_{3}\right): \delta 147.19$, $141.74,131.95,130.85,128.22,128.10,127.42,126.24,125.66,125.02,36.57,26.24,25.65$, 22.62, 18.38. HRMS (EI): $\mathrm{C}_{19} \mathrm{H}_{20}\left[\mathrm{M}^{\cdot}\right]^{+}$calcd 248.1565; found 248.1548.

t-Butyl 2,2-dimethyl-3-hydroxyheptanoate (S3). BuLi (1.6 M in hexanes, $13.5 \mathrm{~mL}$, $0.022 \mathrm{~mol})$ was added to a stirred solution of diisopropylamine $(3.1 \mathrm{~mL}, 0.022 \mathrm{~mol})$ in THF $(20$ $\mathrm{mL})$ at $0{ }^{\circ} \mathrm{C}$. The solution was cooled to $-78^{\circ} \mathrm{C}$, and $t$-butyl isobutyrate $(2.90 \mathrm{~g}, 0.02 \mathrm{~mol})$ was added slowly. The mixture was stirred at $-78^{\circ} \mathrm{C}$ for $30 \mathrm{~min}$. Pentanal $(1.90 \mathrm{~g}, 0.21 \mathrm{~mol})$ was added, and the solution was allowed to warm to room temperature. The reaction mixture was added to water, and the mixture was extracted with ether. The ether extracts were combined, washed with brine, and dried over $\mathrm{MgSO}_{4}$. The ether was removed by rotary evaporation, and the resulting oil was chromatographed on silica gel (Hexanes / ethyl acetate: 8/1) to give a clear oil $(3.10 \mathrm{~g}, 67 \%)$. ${ }^{1} \mathrm{H}-\mathrm{NMR}\left(\mathrm{CDCl}_{3}\right) \delta 0.91(\mathrm{t}, J=7.0 \mathrm{~Hz}, 3 \mathrm{H}), 1.21(\mathrm{~s}, 3 \mathrm{H}), 1.15(\mathrm{~s}, 3 \mathrm{H})$, $1.25-1.42(\mathrm{~m}, 4 \mathrm{H}), 1.46(\mathrm{~s}, 9 \mathrm{H}), 1.48-1.63(\mathrm{~m}, 2 \mathrm{H}), 2.50(\mathrm{~d}, J=7.0 \mathrm{~Hz} 1 \mathrm{H}), 3.54(\mathrm{br} \mathrm{t}, J=7.0$ $\mathrm{Hz}, 1 \mathrm{H}) .{ }^{13} \mathrm{C} \mathrm{NMR}\left(\mathrm{CDCl}_{3}\right): \delta 14.00\left(\mathrm{CH}_{3}\right), 20.86\left(\mathrm{CH}_{3}\right), 22.19\left(\mathrm{CH}_{3}\right), 22.68\left(\mathrm{CH}_{2}\right), 28.02$ $\left(\mathrm{CH}_{3}\right), 28.98\left(\mathrm{CH}_{2}\right), 31.64\left(\mathrm{CH}_{2}\right), 47.44(\mathrm{C}), 76.84(\mathrm{CH}), 80.78(\mathrm{C}), 177.00(\mathrm{C})$.

t-Butyl 2,2-dimethyl-3-methanesulfonyloxyheptanoate (S4a). A solution of S3 (1.55 $\mathrm{g}, 0.0067 \mathrm{~mol})$ and triethylamine $(1.40 \mathrm{~mL}, 0.01 \mathrm{~mol})$ in $\mathrm{CH}_{2} \mathrm{Cl}_{2}(30 \mathrm{~mL})$ was cooled to $-5^{\circ} \mathrm{C}$ in an ice- $\mathrm{NaCl}$ bath. Methanesulfonyl chloride $(0.80 \mathrm{~mL}, 0.01 \mathrm{~mol})$ in $\mathrm{CH}_{2} \mathrm{Cl}_{2}(10 \mathrm{~mL})$ was added slowly over $20 \mathrm{~min}$. After $1 \mathrm{hr}$, the reaction mixture was washed with $\mathrm{HCl}(5 \%), \mathrm{NaHCO}_{3}$ (sat. 
aq.), and brine. After drying over $\mathrm{MgSO}_{4}$, the solvent was removed by rotary evaporation. The resulting oil was chromatographed on silica gel (petroleum ether / ethyl acetate: $5 / 1$ ) to give a clear oil $(1.08 \mathrm{~g}, 52 \%)$. ${ }^{1} \mathrm{H} \mathrm{NMR}\left(\mathrm{CDCl}_{3}\right): \delta 0.89(\mathrm{t}, J=7.0 \mathrm{~Hz}, 3 \mathrm{H}), 1.11(\mathrm{~s}, 3 \mathrm{H}), 1.23(\mathrm{~s}, 3$ H), 1.25-1.42 (m, 3 H), $1.46(\mathrm{~s}, 9 \mathrm{H}), 1.48-1.73(\mathrm{~m}, 3 \mathrm{H}), 3.04(\mathrm{~s}, 3 \mathrm{H}), 4.96(\mathrm{dd}, J=8.8,2.3 \mathrm{~Hz}$, $1 \mathrm{H}) .{ }^{13} \mathrm{C} \mathrm{NMR}\left(\mathrm{CDCl}_{3}\right): \delta 13.86\left(\mathrm{CH}_{3}\right), 19.34\left(\mathrm{CH}_{3}\right), 22.41\left(\mathrm{CH}_{2}\right), 23.25\left(\mathrm{CH}_{3}\right), 27.88\left(\mathrm{CH}_{3}\right)$, $28.52\left(\mathrm{CH}_{2}\right), 31.37\left(\mathrm{CH}_{2}\right), 38.92\left(\mathrm{CH}_{3}\right), 47.61(\mathrm{C}), 81.20(\mathrm{C}), 87.45(\mathrm{CH}), 174.30(\mathrm{C})$. HRMS (ESI): $[\mathrm{M}+\mathrm{Na}]^{+}$; Calc for $\mathrm{C}_{14} \mathrm{H}_{28} \mathrm{SO}_{5} \mathrm{Na}, 331.1555$; found, 331.1555.

t-Butyl 2,2-dimethyl-3-diphenylphosphatoxyheptanoate (S4b). BuLi (8.5 mL, 0.012 mol) was added to a stirred solution of diisopropylamine $(1.7 \mathrm{~mL}, 0.012 \mathrm{~mol})$ in THF at $0{ }^{\circ} \mathrm{C}$. After cooling to $-78{ }^{\circ} \mathrm{C}, t$-butyl isobutyrate $(1.48 \mathrm{~g}, 0.01 \mathrm{~mol})$ was added, and the mixture was stirred for $45 \mathrm{~min}$ at $-78{ }^{\circ} \mathrm{C}$. Pentanal $(0.096 \mathrm{~g}, 0.011 \mathrm{~mol})$ was added. After $5 \mathrm{~min}$, diphenyl chlorophosphate $(2.5 \mathrm{~mL}, 0.012 \mathrm{~mol})$ was added, and the mixture was allowed to warm slowly to room temperature with stirring over $18 \mathrm{~h}$. Water was added to the reaction mixture, and the resulting mixture was extracted with ether. The ether extracts were washed with brine and dried over $\mathrm{MgSO}_{4}$. The resulting oil was chromatographed on silica gel (hexanes / ethyl acetate: $4 / 1$ ) to give a clear oil $(2.05 \mathrm{~g}, 44 \%)$. ${ }^{1} \mathrm{H}-\mathrm{NMR}\left(\mathrm{CDCl}_{3}\right): \delta 0.82(\mathrm{t}, J=7.5 \mathrm{~Hz}, 3 \mathrm{H}), 1.11(\mathrm{~s}, 3 \mathrm{H})$, $1.21(\mathrm{~s}, 3 \mathrm{H}), 1.24-1.40(\mathrm{~m}, 3 \mathrm{H}), 1.43(\mathrm{~s} 9 \mathrm{H}), 1.40-1.52(\mathrm{~m}, 2 \mathrm{H}), 1.61-1.68(\mathrm{~m}, 1 \mathrm{H}), 4.86(\mathrm{td}, J$ $=8.5,1.5 \mathrm{~Hz}, 1 \mathrm{H}), 7.16(\mathrm{t}, J=7.2 \mathrm{~Hz}, 2 \mathrm{H}) 7.16(\mathrm{t}, J=7.5 \mathrm{~Hz}, 4 \mathrm{H}), 7.31(\mathrm{t}, J=7.7 \mathrm{~Hz}, 4 \mathrm{H})$.

${ }^{13} \mathrm{C} \mathrm{NMR}\left(\mathrm{CDCl}_{3}\right): \delta 13.81\left(\mathrm{CH}_{3}\right), 18.42\left(\mathrm{CH}_{3}\right), 22.42\left(\mathrm{CH}_{2}\right), 23.60\left(\mathrm{CH}_{3}\right), 27.84\left(\mathrm{CH}_{3}\right), 28.40$ $\left(\mathrm{CH}_{2}\right), 32.18\left(\mathrm{~d}, J_{\mathrm{C}-\mathrm{P}}=3.1 \mathrm{~Hz}, \mathrm{CH}_{2}\right),\left(48.02, \mathrm{~d}, J_{\mathrm{C}-\mathrm{P}}=5.2 \mathrm{~Hz}, \mathrm{C}\right), 80.89(\mathrm{C}), 86.15\left(\mathrm{~d}, J_{\mathrm{C}-\mathrm{P}}=7.0\right.$ $\mathrm{Hz}, \mathrm{CH}), 120.11\left(\mathrm{~d}, J_{\mathrm{C}-\mathrm{P}}=5.0 \mathrm{~Hz}, \mathrm{CH}\right), 120.14\left(\mathrm{~d}, J_{\mathrm{C}-\mathrm{P}}=5.0 \mathrm{~Hz}, \mathrm{CH}\right), 125.14\left(\mathrm{~d}, J_{\mathrm{C}-\mathrm{P}}=1.0 \mathrm{~Hz}\right.$, $\mathrm{CH}), 125.15\left(\mathrm{~d}, J_{\mathrm{C}-\mathrm{P}}=1.0 \mathrm{~Hz}, \mathrm{CH}\right), 129.66\left(\mathrm{~d}, J_{\mathrm{C}-\mathrm{P}}=1.0 \mathrm{~Hz}, \mathrm{CH}\right), 129.67\left(\mathrm{~d}, J_{\mathrm{C}-\mathrm{P}}=1.0 \mathrm{~Hz}, \mathrm{CH}\right)$, $150.76\left(\mathrm{~d}, J_{\mathrm{C}-\mathrm{P}}=7.3 \mathrm{~Hz}, \mathrm{C}\right), 150.78\left(\mathrm{~d}, J_{\mathrm{C}-\mathrm{P}}=7.3 \mathrm{~Hz}, \mathrm{C}\right), 174.45\left(\mathrm{~d}, J_{\mathrm{C}-\mathrm{P}}=0.9 \mathrm{~Hz}, \mathrm{C}\right)$. HRMS (ESI): $[\mathrm{M}+\mathrm{Na}]^{+}$; Calc for $\mathrm{C}_{25} \mathrm{H}_{35} \mathrm{PO}_{6} \mathrm{Na}$, 485.2069; found, 485.2055.

t-Butyl 2,2-dimethyl-3-methoxyheptanoate (S4c). S3 (1.3 g, 0.0056) was added to a stirred suspension of $\mathrm{NaH}(60 \%$ in mineral oil, $0.3 \mathrm{~g}, 0.0075 \mathrm{~mol})$ in THF $(15 \mathrm{~mL})$. Iodomethane $(1.5 \mathrm{~g}, 0.011 \mathrm{~mol})$ was added, and the mixture was stirred for $18 \mathrm{~h}$. Water was added, and the mixture was extracted with diethyl ether. The combined ether extract was washed with brine and dried over $\mathrm{MgSO}_{4}$. After solvent removal by rotary evaporation, the resulting oil was chromatographed on silica gel (hexanes / ethyl acetate: $10 / 1)$ to give a clear oil $(1.15 \mathrm{~g}, 83 \%)$. ${ }^{1} \mathrm{H}-\mathrm{NMR}\left(\mathrm{CDCl}_{3}\right): \delta 0.91(\mathrm{t}, J=7.0 \mathrm{~Hz}, 3 \mathrm{H}), 1.03(\mathrm{~s}, 3 \mathrm{H}), 1.15(\mathrm{~s}, 3 \mathrm{H}), 1.25-1.40(\mathrm{~m}, 4 \mathrm{H})$, $1.44(\mathrm{~s}, 9 \mathrm{H}), 1.48-1.63(\mathrm{~m}, 2 \mathrm{H}), 3.32(\mathrm{dd}, J=8.5,2.5 \mathrm{~Hz}, 1 \mathrm{H}), 3.45(\mathrm{~s}, 3 \mathrm{H}) .{ }^{13} \mathrm{C} \mathrm{NMR}$ $\left(\mathrm{CDCl}_{3}\right): \delta 14.08,20.25,21.53,23.01,27.98,29.64,31.67,48.32,61.12,79.89,86.60,176.33$.

2,2-dimethyl-3-methanesulfonyloxyheptanoic acid (S5a). A solution of S4a (1.08 g, $0.0035 \mathrm{~mol})$ and trifluoroacetic acid $(2.0 \mathrm{~g}, 0.017 \mathrm{~mol})$ in $\mathrm{CH}_{2} \mathrm{Cl}_{2}(25 \mathrm{~mL})$ was stirred at room temperature for $18 \mathrm{~h}$. The solution was washed with water $(3 \mathrm{x})$, and brine $(3 \mathrm{x})$ and dried over $\mathrm{MgSO}_{4}$. The solvent was removed by rotary evaporation to give a white solid $(0.78 \mathrm{~g}, 87 \%, \mathrm{mp}$ $\left.69-71{ }^{\circ} \mathrm{C}\right) .{ }^{1} \mathrm{H}$ NMR $\left(\mathrm{CDCl}_{3}\right): \delta 0.89(\mathrm{t}, J=7.5 \mathrm{~Hz}, 3 \mathrm{H}), 1.23(\mathrm{~s}, 3 \mathrm{H}), 1.31(\mathrm{~s}, 3 \mathrm{H}), 1.33-1.47$ $(\mathrm{m}, 2 \mathrm{H}), 1.50-1.61(\mathrm{~m}, 2 \mathrm{H}), 1.65-1.78(\mathrm{~m}, 2 \mathrm{H}), 3.04(\mathrm{~s}, 3 \mathrm{H}), 4.99(\mathrm{dd}, J=9.3,2.3 \mathrm{~Hz}, 1 \mathrm{H})$, $11.20($ br s, $1 \mathrm{H}) .{ }^{13} \mathrm{C} \mathrm{NMR}\left(\mathrm{CDCl}_{3}\right): \delta 13.83\left(\mathrm{CH}_{3}\right), 20.51\left(\mathrm{CH}_{3}\right), 21.71\left(\mathrm{CH}_{3}\right), 22.35\left(\mathrm{CH}_{2}\right)$, $28.37\left(\mathrm{CH}_{2}\right), 30.81\left(\mathrm{CH}_{2}\right), 38.90\left(\mathrm{CH}_{3}\right), 47.09(\mathrm{C}), 86.91(\mathrm{CH}), 181.70(\mathrm{C})$. HRMS (ESI): [M + $\mathrm{Na}]^{+}$; Calc for $\mathrm{C}_{10} \mathrm{H}_{20} \mathrm{SO}_{5} \mathrm{Na}, 275.0929$; found, 275.0921 .

2,2-dimethyl-3-diphenylphosphatoxyheptanoic acid (S5b). A solution of $\mathbf{S 4 b}(2.0 \mathrm{~g}$, $0.0043 \mathrm{~mol})$ and trifluoroacetic acid $(6.1 \mathrm{~g}, 0.05 \mathrm{~mol})$ in $\mathrm{CH}_{2} \mathrm{Cl}_{2}(30 \mathrm{~mL})$ was stirred at room temperature for $16 \mathrm{~h}$. The solution was washed with water $(3 \mathrm{x})$ and brine $(3 \mathrm{x})$ and dried over $\mathrm{MgSO}_{4}$. The solvent was removed by rotary evaporation to give a viscous oil $(1.39 \mathrm{~g}, 79 \%)$. ${ }^{1} \mathrm{H}$ $\operatorname{NMR}\left(\mathrm{CDCl}_{3}\right): \delta 0.79(\mathrm{t}, J=7.2 \mathrm{~Hz}, 3 \mathrm{H}), 1.15(\mathrm{~s}, 3 \mathrm{H}), 1.23(\mathrm{~s}, 3 \mathrm{H}), 1.20-1.68(\mathrm{~m}, 6 \mathrm{H}), 4.89$ $(\mathrm{td}, J=8.5,2.0 \mathrm{~Hz}, 1 \mathrm{H}), 7.12-7.38(\mathrm{~m}, 10 \mathrm{H}) .{ }^{13} \mathrm{C} \mathrm{NMR}\left(\mathrm{CDCl}_{3}\right) \delta 13.91\left(\mathrm{CH}_{3}\right), 19.72\left(\mathrm{CH}_{3}\right)$, $22.21\left(\mathrm{CH}_{3}\right), 22.47\left(\mathrm{CH}_{2}\right), 28.28\left(\mathrm{CH}_{2}\right), 31.71\left(\mathrm{~d}, J_{\mathrm{C}-\mathrm{P}}=3.4 \mathrm{~Hz}, \mathrm{CH}_{2}\right), 47.40\left(\mathrm{~d}, J_{\mathrm{C}-\mathrm{P}}=4.9 \mathrm{~Hz}\right.$, C), $85.92\left(\mathrm{~d}, J_{\mathrm{C}-\mathrm{P}}=7.2 \mathrm{~Hz}, \mathrm{CH}\right), 120.04(\mathrm{CH}), 120.11(\mathrm{CH}), 125.13\left(\mathrm{~d}, J_{\mathrm{C}-\mathrm{P}}=1.3 \mathrm{~Hz}, \mathrm{CH}\right)$, 
$125.16\left(\mathrm{~d}, J_{\mathrm{C}-\mathrm{P}}=1.3 \mathrm{~Hz}, \mathrm{CH}\right), 129.56\left(\mathrm{~d}, J_{\mathrm{C}-\mathrm{P}}=1.0 \mathrm{~Hz}, \mathrm{CH}\right), 129.57\left(\mathrm{~d}, J_{\mathrm{C}-\mathrm{P}}=1.0 \mathrm{~Hz}, \mathrm{CH}\right)$, $150.48(\mathrm{C}), 150.57(\mathrm{C}), 180.48\left(\mathrm{~d}, J_{\mathrm{C}-\mathrm{P}}=0.5 \mathrm{~Hz}, \mathrm{C}\right)$. HRMS (ESI): $[\mathrm{M}+\mathrm{Na}]^{+}$; Calc for $\mathrm{C}_{21} \mathrm{H}_{27} \mathrm{PO}_{6} \mathrm{Na}$, 429.1433; found, 429.1429.

2,2-dimethyl-3-methoxyheptanoic acid (S5c). A solution of S4c (1.15 g, $0.0047 \mathrm{~mol})$ and trifluoroacetic acid $(4.5 \mathrm{~g}, 0.039 \mathrm{~mol})$ in $\mathrm{CH}_{2} \mathrm{Cl}_{2}(30 \mathrm{~mL})$ was stirred at room temperature for $16 \mathrm{~h}$. The reaction mixture was washed with water and brine and dried over $\mathrm{MgSO}_{4}$ to give a viscous oil $(0.82 \mathrm{~g}, 93 \%)$. ${ }^{1} \mathrm{H}$ NMR $\left(\mathrm{CDCl}_{3}\right): \delta 0.91(\mathrm{t}, J=7.1 \mathrm{~Hz}, 3 \mathrm{H}), 1.15(\mathrm{~s}, 3 \mathrm{H}), 1.20(\mathrm{~s}, 3$ $\mathrm{H}), 1.25-1.50(\mathrm{~m}, 6 \mathrm{H}), 3.36(\mathrm{dd}, J=7.2,4.7 \mathrm{~Hz}, 1 \mathrm{H}), 3.48(\mathrm{~s}, 3 \mathrm{H}) 11.2 \mathrm{br} \mathrm{s}, 1 \mathrm{H}) .{ }^{13} \mathrm{C} \mathrm{NMR}$ $\left(\mathrm{CDCl}_{3}\right): \delta 13.98\left(\mathrm{CH}_{3}\right), 20.87\left(\mathrm{CH}_{3}\right), 21.27\left(\mathrm{CH}_{3}\right), 22.89\left(\mathrm{CH}_{2}\right), 29.26\left(\mathrm{CH}_{2}\right), 31.26\left(\mathrm{CH}_{2}\right)$, 47.58, $61.00\left(\mathrm{CH}_{3}\right), 86.62(\mathrm{CH}), 182.96(\mathrm{C})$. HRMS (ESI): $[\mathrm{M}+\mathrm{Na}]^{+}$; Calc for $\mathrm{C}_{10} \mathrm{H}_{20} \mathrm{O}_{3} \mathrm{Na}$, 211.1310; found, 211.1320.

(1H)-Thioxo-1-pyridyl 2,2-dimethyl-3-methanesulfonyloxyheptanoate (1a). A solution of S5a $(0.71 \mathrm{~g}, 0.0028 \mathrm{~mol})$ and 2,2'-dithiopyridene-1-1'-(bis)- $N$-oxide $(1.06 \mathrm{~g}, 0.0042$ mol) in $\mathrm{CH}_{2} \mathrm{Cl}_{2}(15 \mathrm{~mL})$ was cooled to $0{ }^{\circ} \mathrm{C}$ and shielded from light. Tributylphosphine $(1.0 \mathrm{~mL}$, $0.0042 \mathrm{~mol}$ ) was added, and the mixture was stirred for $1.5 \mathrm{~h}$. The reaction mixture was washed with $\mathrm{NaHCO}_{3}$ (sat. aq) and brine. The solution was dried over $\mathrm{MgSO}_{4}$, and the solvent was removed by rotary evaporation. The resulting oil was chromatographed on silica gel (hexanes / ethyl acetate: $1 / 1)$ to give a yellow solid $\left(0.66 \mathrm{~g}, 65 \%, \mathrm{mp}=73-76^{\circ} \mathrm{C}\right) .{ }^{\mathrm{l}} \mathrm{H} \mathrm{NMR}\left(\mathrm{CDCl}_{3}\right): \delta$ $0.93(\mathrm{t}, J=7.2 \mathrm{~Hz}, 3 \mathrm{H}), 1.49(\mathrm{~s}, 3 \mathrm{H}), 1.55(\mathrm{~s}, 3 \mathrm{H}), 1.33-1.47(\mathrm{~m}, 4 \mathrm{H}), 1.70-1.82(\mathrm{~m}, 2 \mathrm{H})$, $3.08(\mathrm{~s}, 3 \mathrm{H}), 5.14(\mathrm{dd}, J=9.0,3.2 \mathrm{~Hz}, 1 \mathrm{H}), 6.40(\mathrm{td}, J=7.0,1.8 \mathrm{~Hz}, 1 \mathrm{H}), 7.20$ (ddd, $J=8.5$, 7.0, $1.6 \mathrm{~Hz}, 1 \mathrm{H}), 7.6(\mathrm{dd}, J=8.8,1.4 \mathrm{~Hz}, 1 \mathrm{H}), 7.83(\mathrm{dd}, J=7.1,1.3 \mathrm{~Hz}, 1 \mathrm{H}) .{ }^{13} \mathrm{C} \mathrm{NMR}$ $\left(\mathrm{CDCl}_{3}\right): \delta 13.89\left(\mathrm{CH}_{3}\right), 19.70\left(\mathrm{CH}_{3}\right), 22.29\left(\mathrm{CH}_{3}\right), 22.33\left(\mathrm{CH}_{2}\right), 28.39\left(\mathrm{CH}_{2}\right), 29.80\left(\mathrm{CH}_{2}\right)$, $39.05\left(\mathrm{CH}_{3}\right), 47.54(\mathrm{C}), 88.88(\mathrm{CH}), 112.80(\mathrm{CH}), 133.75(\mathrm{CH}), 137.23(\mathrm{CH}), 138.19(\mathrm{CH})$, $170.80(\mathrm{C}), 175.86(\mathrm{C})$. HRMS (ESI): $[\mathrm{M}+\mathrm{H}]^{+}$; calc for $\mathrm{C}_{15} \mathrm{H}_{24} \mathrm{~S}_{2} \mathrm{NO}_{5}, 362.1096$; found, 362.1104 .

(1H)-Thioxo-1-pyridyl 2,2-dimethyl-3-diphenylphosphatoxyheptanoate (1b). A solution of S5b (1.30 g, $0.0031 \mathrm{~mol})$ and 2,2'-dithiopyridene-1-1'-(bis)- $N$-oxide (1.20 g, 0.0047 mol) in $\mathrm{CH}_{2} \mathrm{Cl}_{2}(20 \mathrm{~mL})$ was cooled to $0^{\circ} \mathrm{C}$ and shielded from light. Tributylphosphine $(1.1 \mathrm{~mL}$, $0.0044 \mathrm{~mol}$ ) was added, and the mixture was stirred for $1.5 \mathrm{~h}$. The reaction mixture was washed with $\mathrm{NaHCO}_{3}$ (sat. aq) and brine. The solution was dried over $\mathrm{MgSO}_{4}$, and the solvent was removed by rotary evaporation. The resulting oil was chromatographed on silica gel (hexanes / ethyl acetate: $1 / 1)$ to give a yellow oil $(1.1 \mathrm{~g}, 65 \%) .{ }^{1} \mathrm{H}-\mathrm{NMR}\left(\mathrm{CDCl}_{3}\right): \delta 0.85(\mathrm{t}, J=7.2 \mathrm{~Hz}, 3$ $\mathrm{H}), 1.48(\mathrm{~s}, 3 \mathrm{H}), 1.56(\mathrm{~s}, 3 \mathrm{H}), 1.20-1.57(\mathrm{~m}, 4 \mathrm{H}), 1.64-1.80(\mathrm{~m}, 2 \mathrm{H}), 5.05$ (td, $J=8.1,3.6 \mathrm{~Hz}$, $1 \mathrm{H}), 6.42$ (t, $J=6.0 \mathrm{~Hz}, 1 \mathrm{H}), 7.03-7.40(\mathrm{~m}, 11 \mathrm{H}), 7.64(\mathrm{~d}, J=8.4 \mathrm{~Hz}, 1 \mathrm{H}), 7.89$ (d, $J=6.9 \mathrm{~Hz}$, $1 \mathrm{H}) .{ }^{13} \mathrm{C}$ NMR (benzene-d $): \delta 14.06\left(\mathrm{CH}_{3}\right), 18.76\left(\mathrm{CH}_{3}\right), 22.59\left(\mathrm{CH}_{3}\right), 22.82\left(\mathrm{CH}_{2}\right), 28.49$ $\left(\mathrm{CH}_{2}\right), 30.40\left(\mathrm{~d}, J_{\mathrm{C}-\mathrm{P}}=3.6 \mathrm{~Hz}, \mathrm{CH}_{2}\right), 48.14\left(\mathrm{~d}, J_{\mathrm{C}-\mathrm{P}}=3.9 \mathrm{~Hz}, \mathrm{C}\right), 85.70\left(\mathrm{~d}, J_{\mathrm{C}-\mathrm{P}}=6.7 \mathrm{~Hz}, \mathrm{CH}\right)$, $111.19(\mathrm{CH}), 120.39\left(\mathrm{~d}, J_{\mathrm{C}-\mathrm{P}}=5.1 \mathrm{~Hz}, \mathrm{CH}\right), 120.71\left(\mathrm{~d}, J_{\mathrm{C}-\mathrm{P}}=4.7 \mathrm{~Hz}, \mathrm{CH}\right), 125.45(\mathrm{CH}), 125.53$ $(\mathrm{CH}), 129.91(\mathrm{CH}), 129.99(\mathrm{CH}), 132.60(\mathrm{CH}), 137.22(\mathrm{CH}), 138.73(\mathrm{CH}), 151.10\left(\mathrm{~d}, J_{\mathrm{C}-\mathrm{P}}=7.3\right.$ $\mathrm{Hz}, \mathrm{C}), 151.26$ (d, $\left.J_{\mathrm{C}-\mathrm{P}}=7.0 \mathrm{~Hz}, \mathrm{C}\right), 171.22(\mathrm{C}), 176.81(\mathrm{C})$. HRMS (ESI): $\left[\mathrm{M}+\mathrm{Na}^{+}\right.$; Calc for $\mathrm{C}_{26} \mathrm{H}_{30} \mathrm{PSNO}_{6} \mathrm{Na}, 538.1429$; found, 538.1440.

(1H)-Thioxo-1-pyridyl 2,2-dimethyl-3-methoxyheptanoate (1c). A solution of S5c (0.82 g, $0.0043 \mathrm{~mol})$ and 2,2'-dithiopyridene-1-1'-(bis)- $N$-oxide (1.65 g, $0.0065 \mathrm{~mol})$ in $\mathrm{CH}_{2} \mathrm{Cl}_{2}$ $(10 \mathrm{~mL})$ was cooled to $0^{\circ} \mathrm{C}$ and shielded from light. Tributylphosphine $(1.8 \mathrm{~mL}, 0.0065 \mathrm{~mol})$ was added, and the mixture was stirred for $1.5 \mathrm{~h}$. The reaction mixture was washed with $\mathrm{NaHCO}_{3}$ (sat. aq) and brine. The solution was dried over $\mathrm{MgSO}_{4}$, and the solvent was removed by rotary evaporation. The resulting oil was chromatographed on silica gel (hexanes / ethyl acetate: $1 / 1)$ to give a yellow solid $\left(0.60 \mathrm{~g}, 46 \%, \mathrm{mp}=39-41{ }^{\circ} \mathrm{C}\right) .{ }^{1} \mathrm{H} \mathrm{NMR}\left(\mathrm{CDCl}_{3}\right): \delta 0.92(\mathrm{t}$, $J=7.1 \mathrm{~Hz}, 3 \mathrm{H}), 1.25-1.50(\mathrm{~m}, 3 \mathrm{H}), 1.41(\mathrm{~s}, 3 \mathrm{H}), 1.43(\mathrm{~s}, 3 \mathrm{H}), 1.50-1.63(\mathrm{~m}, 3 \mathrm{H}), 3.50$ (singlet superimposed on multiplet, $4 \mathrm{H}), 6.63(\mathrm{td}, J=7.0,1.8 \mathrm{~Hz}, 1 \mathrm{H}), 7.19$ (ddd, $J=8.6,6.8$, $1.6 \mathrm{~Hz}, 1 \mathrm{H}), 7.46(\mathrm{dd}, J=7.0,1.1 \mathrm{~Hz}, 1 \mathrm{H}), 7.65(\mathrm{dd}, J=8.8,1.6 \mathrm{~Hz}, 1 \mathrm{H}) .{ }^{13} \mathrm{C} \mathrm{NMR}\left(\mathrm{CDCl}_{3}\right)$ : 


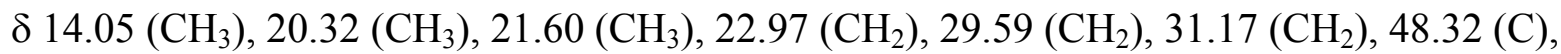
$61.85\left(\mathrm{CH}_{3}\right), 86.32(\mathrm{CH}), 112.48(\mathrm{CH}), 133.20(\mathrm{CH}), 137.43(\mathrm{CH}), 137.65(\mathrm{CH}), 172.22(\mathrm{C})$, 175.95 (C). HRMS (ESI): $[\mathrm{M}+\mathrm{Na}]^{+}$; calc for $\mathrm{C}_{15} \mathrm{H}_{23} \mathrm{NO}_{3} \mathrm{SNa}, 320.1287$; found, 320.1288 .

3-Methanesulfonyloxy-2-methylheptane (S6a). A solution of 2-methylheptan-3-ol) $(1.50 \mathrm{~g}, 0.011 \mathrm{~mol})$ and triethylamine $(2.4 \mathrm{~mL}, 0.016 \mathrm{~mol})$ in $\mathrm{CH}_{2} \mathrm{Cl}_{2}(40 \mathrm{~mL})$ was cooled to -5 ${ }^{\circ} \mathrm{C}$ in an ice- $\mathrm{NaCl}$ bath. Methanesulfonyl chloride $(1.30 \mathrm{~mL}, 0.016 \mathrm{~mol})$ in $\mathrm{CH}_{2} \mathrm{Cl}_{2}(10 \mathrm{~mL})$ was added slowly over 20 min. After $1.5 \mathrm{~h}$, the reaction mixture was washed with $\mathrm{HCl}(5 \%)$, $\mathrm{NaHCO}_{3}$ (sat. aq.), and brine. After drying over $\mathrm{MgSO}_{4}$, the solvent was removed by rotary evaporation. The resulting oil was chromatographed on silica gel (petroleum ether / ethyl acetate: $5 / 1)$ to give a clear oil $(1.80 \mathrm{~g}, 75 \%)$. ${ }^{1} \mathrm{H}$ NMR $\left(\mathrm{CDCl}_{3}\right): \delta 0.84-1.02\left(\mathrm{~m}_{13} 9 \mathrm{H}\right), 0.088$ $(\mathrm{s}, 3 \mathrm{H}), 1.37(\mathrm{~m}, \mathrm{H}), 1.67(\mathrm{~m}, 2 \mathrm{H}), 2.04(\mathrm{~m}, 1 \mathrm{H}), 3.01(\mathrm{~s}, 3 \mathrm{H}), 4.56(\mathrm{~m}, 1 \mathrm{H}) .{ }^{13} \mathrm{C} \mathrm{NMR}$ $\left(\mathrm{CDCl}_{3}\right): \delta 13.78\left(\mathrm{CH}_{3}\right), 17.26\left(\mathrm{CH}_{3}\right), 17.87\left(\mathrm{CH}_{3}\right), 22.37\left(\mathrm{CH}_{2}\right), 27.21\left(\mathrm{CH}_{2}\right), 30.53\left(\mathrm{CH}_{2}\right)$, $31.24(\mathrm{CH}), 38.57\left(\mathrm{CH}_{3}\right), 88.60(\mathrm{CH})$.

3-(2-methylheptyl)diphenylphosphate (10). BuLi (7.0 mL, 1.4 M, 0.010 mol) was added to a stirred solution of diisopropylamine $(1.7 \mathrm{~mL}, 0.012 \mathrm{~mol})$ in THF $(40 \mathrm{~mL})$ at $0{ }^{\circ} \mathrm{C}$. 2 Methylheptan-3-ol (1.3 g, $0.01 \mathrm{~mol})$ was added. Diphenyl chlorophosphate $(2.2 \mathrm{~mL}, 0.011 \mathrm{~mol})$ was added, and the mixture was stirred at room temperature for $18 \mathrm{~h}$. Water was added, and the mixture was extracted with ether. The ether extracts were washed with brine and dried over $\mathrm{MgSO}_{4}$. After solvent removal by rotary evaporation, the resulting oil was chromatographed on silica gel (hexanes / ethyl acetate: $5 / 1)$ to give a clear oil $(1.65 \mathrm{~g}, 50 \%) .{ }^{1} \mathrm{H}$ NMR $\left(\mathrm{CD}_{3} \mathrm{CN}\right): \delta$ $0.82(\mathrm{t}, J=7.1 \mathrm{~Hz}, 3 \mathrm{H}), 0.088(\mathrm{~s}, 3 \mathrm{H}), 0.90(\mathrm{~s}, 3 \mathrm{H}), 1.28(\mathrm{~m}, 5 \mathrm{H}), 1.60(\mathrm{~m}, \mathrm{H}), 1.95(\mathrm{~m}, 1 \mathrm{H})$, 4.46 (tt, $J=7.4,4.6 \mathrm{~Hz}, 1 \mathrm{H}), 7.19(\mathrm{~m}, 6 \mathrm{H}), 7.21(\mathrm{~m}, 4 \mathrm{H}) .{ }^{13} \mathrm{C} \mathrm{NMR}\left(\mathrm{CD}_{3} \mathrm{CN}\right): \delta 14.23$ $\left(\mathrm{CH}_{3}\right), 17.41\left(\mathrm{CH}_{3}\right), 18.32\left(\mathrm{CH}_{3}\right), 23.14\left(\mathrm{CH}_{2}\right), 27.98\left(\mathrm{CH}_{2}\right), 32.07\left(\mathrm{~d}, J_{\mathrm{C}-\mathrm{P}}=4.1 \mathrm{~Hz}, \mathrm{CH}_{2}\right), 32.62$ $\left(\mathrm{d}, J_{\mathrm{C}-\mathrm{P}}=4.6 \mathrm{~Hz}, \mathrm{CH}\right), 87.69\left(\mathrm{~d}, J_{\mathrm{C}-\mathrm{P}}=7.3 \mathrm{~Hz}, \mathrm{CH}\right), 120.84\left(\mathrm{~d}, J_{\mathrm{C}-\mathrm{P}}=4.6 \mathrm{~Hz}, \mathrm{CH}\right), 120.86\left(\mathrm{~d}, J_{\mathrm{C}-}\right.$ $\mathrm{P}=4.9 \mathrm{~Hz}, \mathrm{CH}), 126.10\left(\mathrm{~d}, J_{\mathrm{C}-\mathrm{P}}=1.0 \mathrm{~Hz}, \mathrm{CH}\right), 126.11\left(\mathrm{~d}, J_{\mathrm{C}-\mathrm{P}}=1.0 \mathrm{~Hz}, \mathrm{CH}\right), 130.64(\mathrm{CH})$, $130.65(\mathrm{CH}), 151.52\left(\mathrm{~d}, J_{\mathrm{C}-\mathrm{P}}=7.3 \mathrm{~Hz}, \mathrm{C}\right), 151.52\left(\mathrm{~d}, J_{\mathrm{C}-\mathrm{P}}=7.0 \mathrm{~Hz}, \mathrm{C}\right)$. HRMS (ESI): [M + $\mathrm{Na}]^{+}$; calc for $\mathrm{C}_{20} \mathrm{H}_{27} \mathrm{PO}_{4} \mathrm{Na}, 385.1545$; found, 385.1537 .

DFT Calculations. B3LYP/6-31G* calculations on 7 and is secondary radical isomer were carried out using Gaussian 98. ${ }^{\mathrm{S} 4}$ The electronic energies and zero point energies for the minimized structures are given in Table $\mathrm{S} 1$.

Table S1. B3LYP/6-31G* calculated energies and zero point corrections in Hartrees.

\begin{tabular}{lrr}
\hline & \multicolumn{1}{c}{7} & \multicolumn{1}{c}{ isomer of 7} \\
\hline electronic energy & -446.95791 & -446.95342 \\
zero-point energy & 0.26988 & 0.36969 \\
sum & -446.68807 & -446.68373 \\
\hline
\end{tabular}


Table S2. Product ratios from photolysis of $\mathbf{1 b}$ in $\mathrm{CD}_{3} \mathrm{CN}$.

\begin{tabular}{cr}
\hline$[\mathrm{PhSH}]_{\text {average }}(\mathrm{M})$ & {$[\mathbf{1 0}] /[\mathbf{1 1}]$} \\
\hline 0.795 & $20.8 \pm 2.9$ \\
0.695 & $17.4 \pm 2.5$ \\
0.59 & $12.4 \pm 1.7$ \\
0.59 & $10.8 \pm 1.5$ \\
0.49 & $10.8 \pm 1.5$ \\
0.39 & $9.8 \pm 1.4$ \\
0.29 & $5.9 \pm 0.8$ \\
0.19 & $3.8 \pm 0.5$ \\
0.09 & $1.7 \pm 0.2$ \\
0.09 & $2.3 \pm 0.3$ \\
0.05 & $2.5 \pm 0.3$ \\
\hline
\end{tabular}

Table S3. Kinetic data from LFP studies of radical 1d.

\begin{tabular}{|c|c|c|c|}
\hline Temp $\left({ }^{\circ} \mathrm{C}\right)$ & $10^{-6} \times k_{\mathrm{obs}}\left(\mathrm{s}^{-1}\right)$ & Temp $\left({ }^{\circ} \mathrm{C}\right)$ & $10^{-6} \times k_{\mathrm{obs}}\left(\mathrm{s}^{-1}\right)$ \\
\hline $\begin{array}{l}50.7 \\
50.5 \\
43.2 \\
43.0 \\
39.5 \\
39.4 \\
31.9 \\
31.9 \\
25.7 \\
25.6 \\
21.9 \\
21.8 \\
19.8 \\
19.7 \\
18.3 \\
18.2 \\
10.3 \\
10.2\end{array}$ & $\begin{array}{c}31.5 \pm 0.3 \\
29.8 \pm 0.2 \\
26.6 \pm 0.1 \\
27.0 \pm 0.3 \\
24.2 \pm 0.1 \\
24.7 \pm 0.2 \\
19.2 \pm 0.2 \\
20.4 \pm 0.1 \\
18.0 \pm 0.1 \\
18.6 \pm 0.1 \\
16.4 \pm 0.1 \\
16.9 \pm 0.1 \\
15.7 \pm 0.1 \\
15.7 \pm 0.1 \\
13.4 \pm 0.1 \\
13.0 \pm 0.1 \\
9.90 \pm 0.05 \\
9.96 \pm 0.04\end{array}$ & $\begin{array}{r}0.6 \\
0.5 \\
-8.8 \\
-8.8 \\
-10.7 \\
-20.5 \\
-20.5 \\
-20.6 \\
-30.8 \\
-30.8 \\
-30.9 \\
-30.9 \\
-38.5 \\
-39.0 \\
-39.1 \\
-39.2\end{array}$ & $\begin{array}{l}6.30 \pm 0.06 \\
6.22 \pm 0.04 \\
4.63 \pm 0.02 \\
4.34 \pm 0.04 \\
5.24 \pm 0.02 \\
3.04 \pm 0.03 \\
2.94 \pm 0.03 \\
3.04 \pm 0.03 \\
1.93 \pm 0.02 \\
1.97 \pm 0.03 \\
1.91 \pm 0.02 \\
2.03 \pm 0.02 \\
1.43 \pm 0.03 \\
1.52 \pm 0.01 \\
1.36 \pm 0.03 \\
1.52 \pm 0.01\end{array}$ \\
\hline $\begin{array}{r}\mathrm{CH}_{3} \mathrm{CN} \\
-4.2 \\
-20.5 \\
-20\end{array}$ & $\begin{array}{l}70.8 \pm 0.8 \\
46.6 \pm 0.3 \\
46.5 \pm 0.3\end{array}$ & $\begin{array}{l}-19 \\
-40\end{array}$ & $\begin{array}{l}51.2 \pm 0.3 \\
24.0 \pm 0.2\end{array}$ \\
\hline
\end{tabular}




\section{References for supporting information}

(S1) Pangborn, A. B.; Giardello, M. A.; Grubbs, R. H.; Rosen, R. K.; Timmers, F. J. Organometallics 1996, 15, 1518-1520.

(S2) Choi, S.-Y.; Crich, D.; Horner, J. H.; Huang, X.; Newcomb, M.; Whitted, P. O. Tetrahedron 1999, 55, 3317-3326.

(S3) Horner, J. H.; Tanaka, N.; Newcomb, M. J. Am. Chem. Soc. 1998, 120, 10379.

(S4) Frish, M. J.; Trucks, G. W.; Schlegel, H. B.; Scuseria, G. E.; Robb, M. A.; Cheeseman, J. R.; Zakrzewski, V. G.; Montgomery, J. A.; Jr.; Stratmann, R. E.; Burant, J. C.; Dapprich, S.; Millam, J. M.; Daniels, A. D.; Kudin, K. N.; Strian, M. C.; Farkas, O.; Tomasi, J.; Barone, V.; Cossi, M.; Cammi, R.; Mennucci, B.; Pomelli, C.; Adamo, C.; Clifford, S.; Ochterski, J.; Petersson, G. A.; Ayala, P. Y.; Cui, P. Y.; Morokuma, K.; Malick, D. K.; Rabuck, A. D.; Raghavachari, K.; Foresman, J. B.; Cioslowski, J.; Ortiz, A. G.; Stefanov, B. B.; Liu, G.; Liashenko, A.; Piskorz, P.; Komaromi, I.; Gomperts, R.; Martin, R. L.; Fox, D. J.; Keith, T.; Al-Laham, M. A.; Peng, C. Y.; Nanayakkara, A.; Gonzalez, C.; Challacombe, M.; Gill, P. M. W.; Johnson, B.; Chen, W.; Wong, M. W.; Andres, J. L.; Gonzalez, C.; Head-Gordon, M.; Replogle, E. S.; Pople, J. A. Gaussian 98 Revision A.3, Gaussian, Inc., Pittsburgh, PA, 1998. 


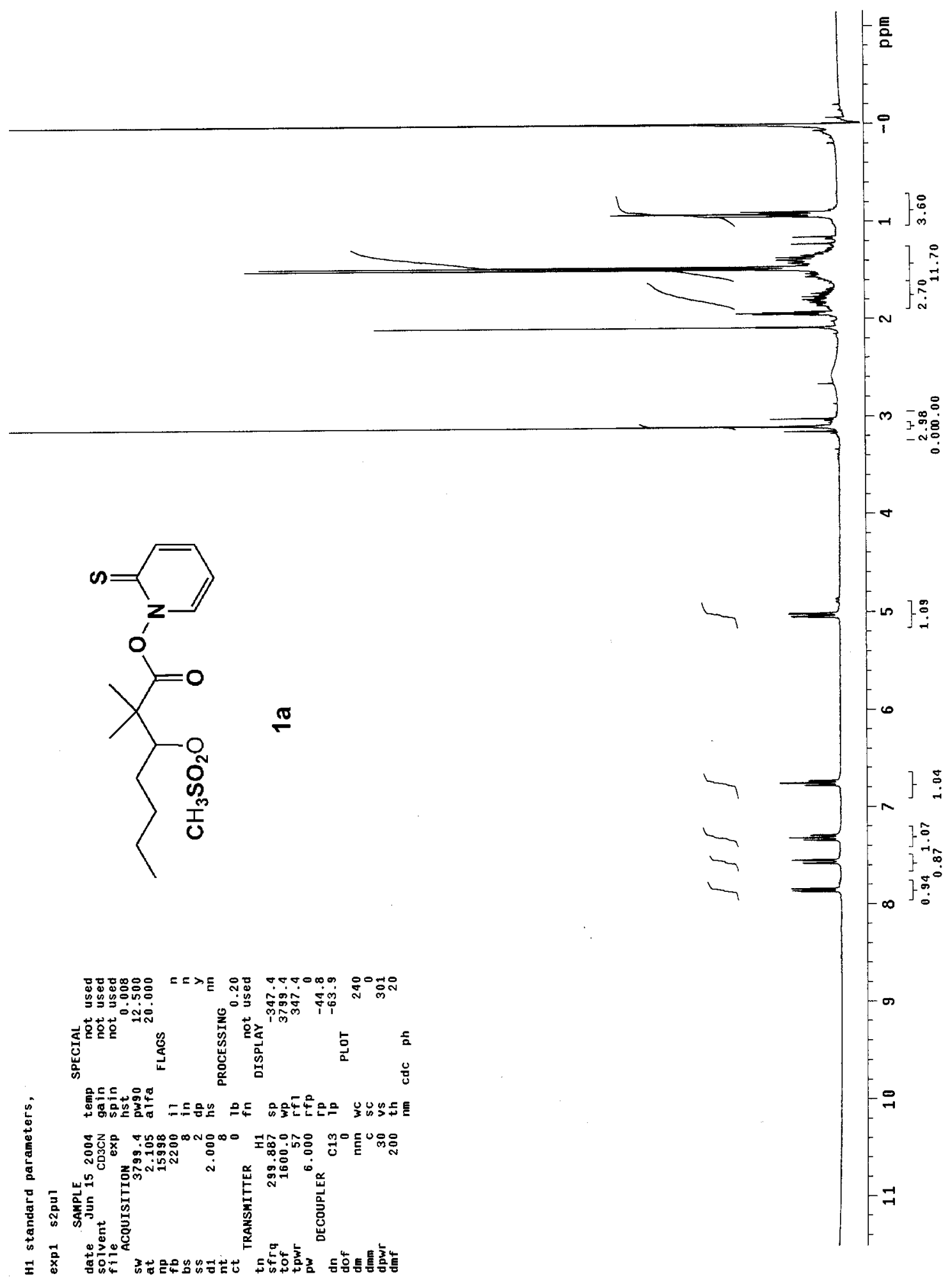




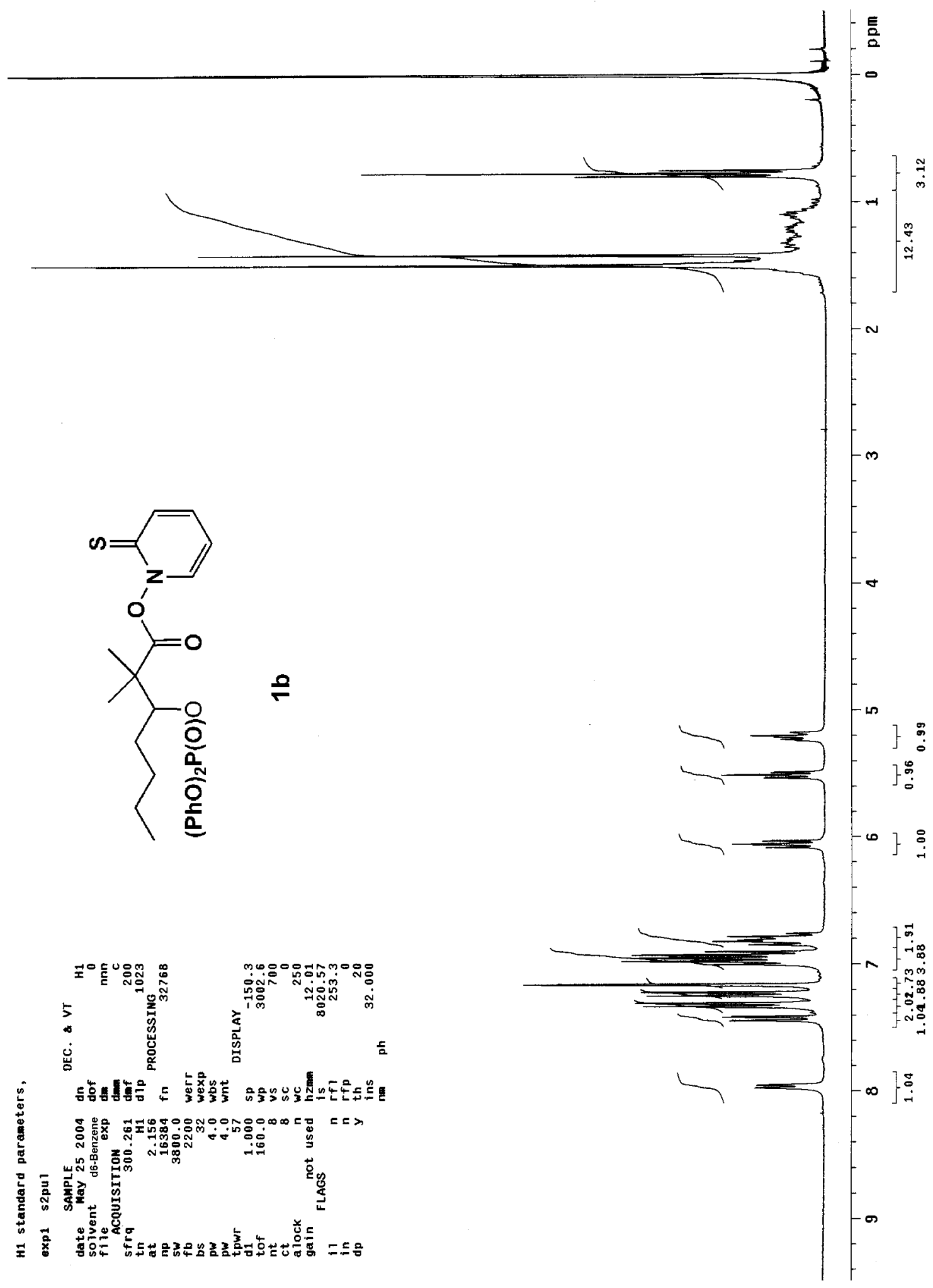




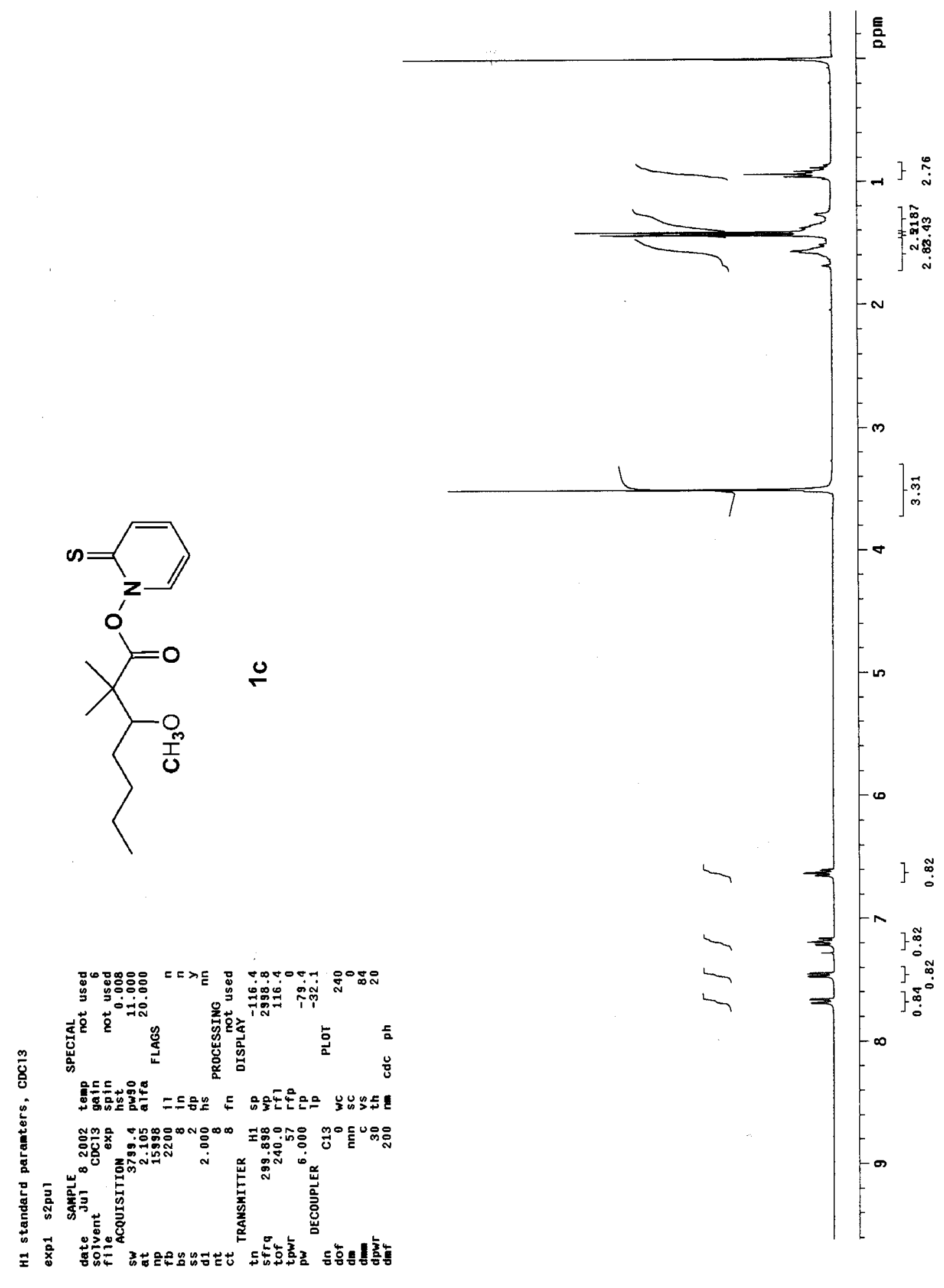




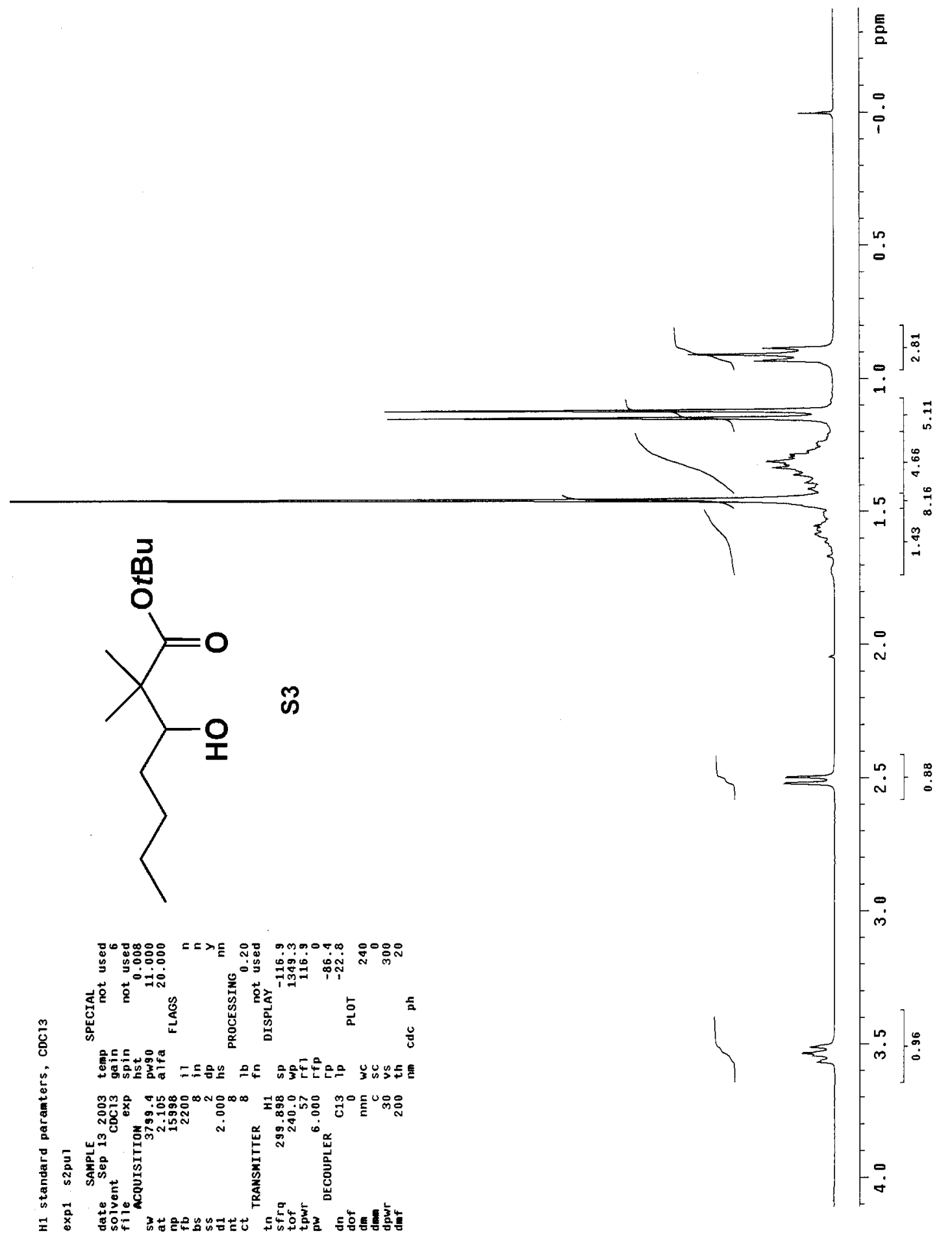




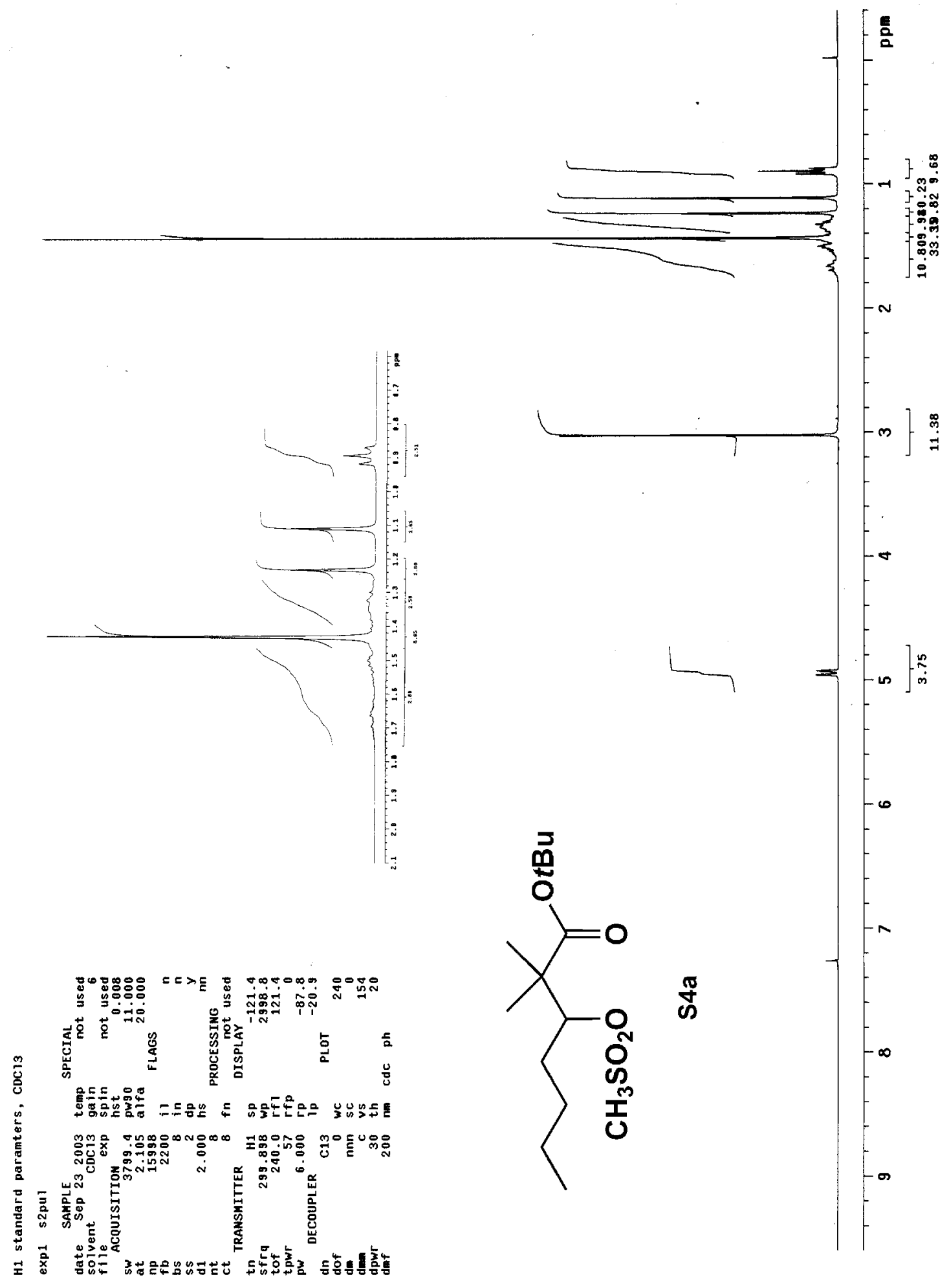



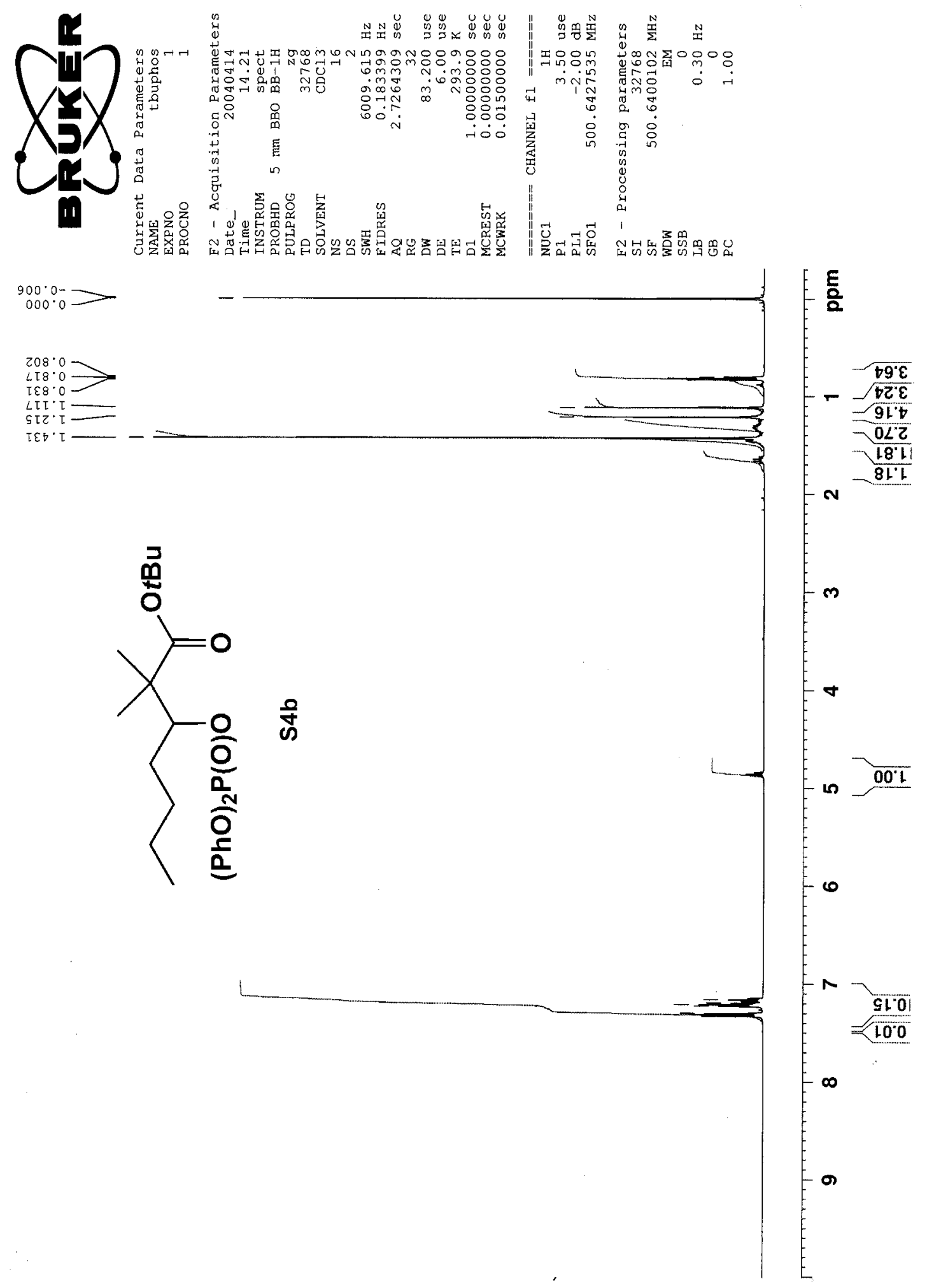


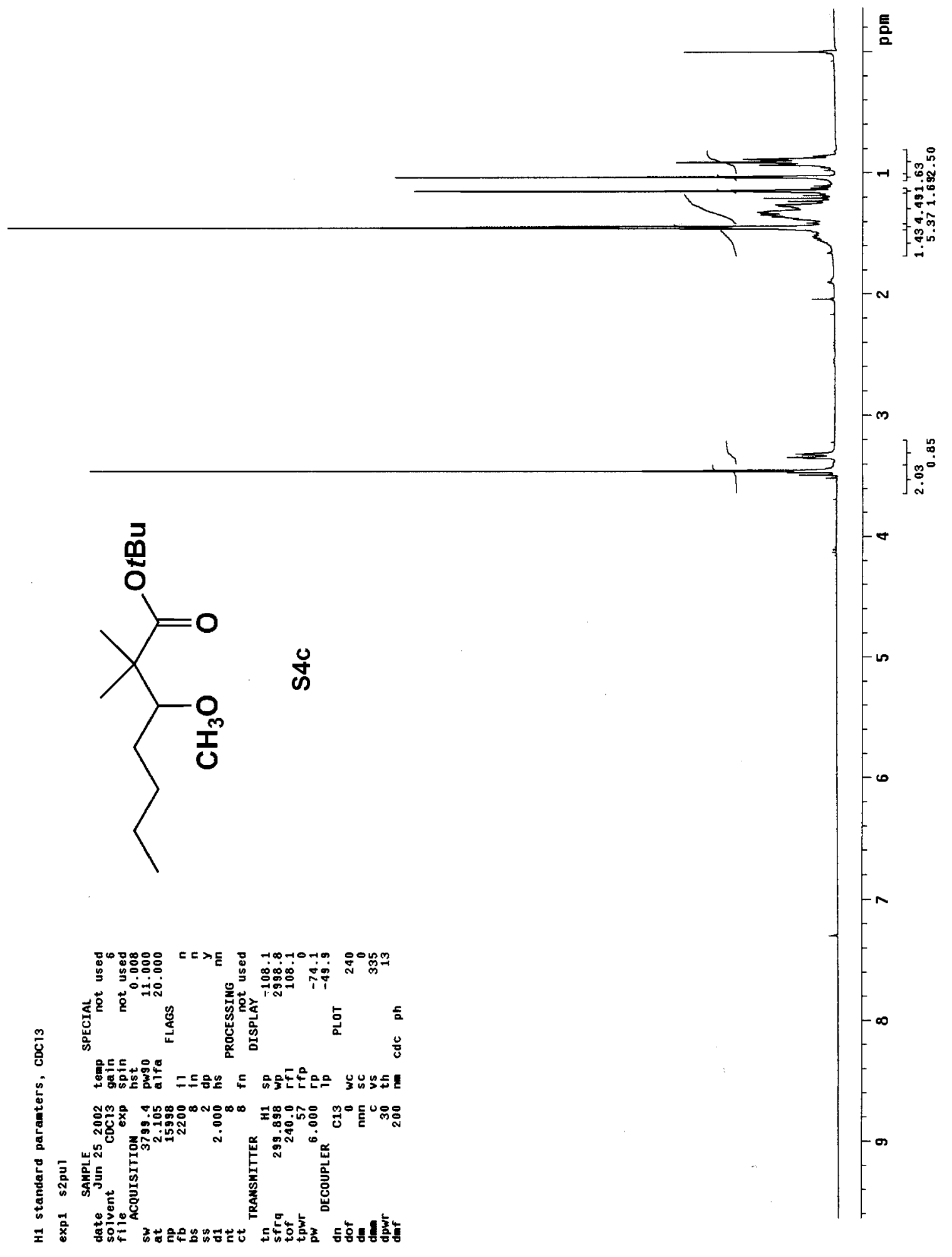




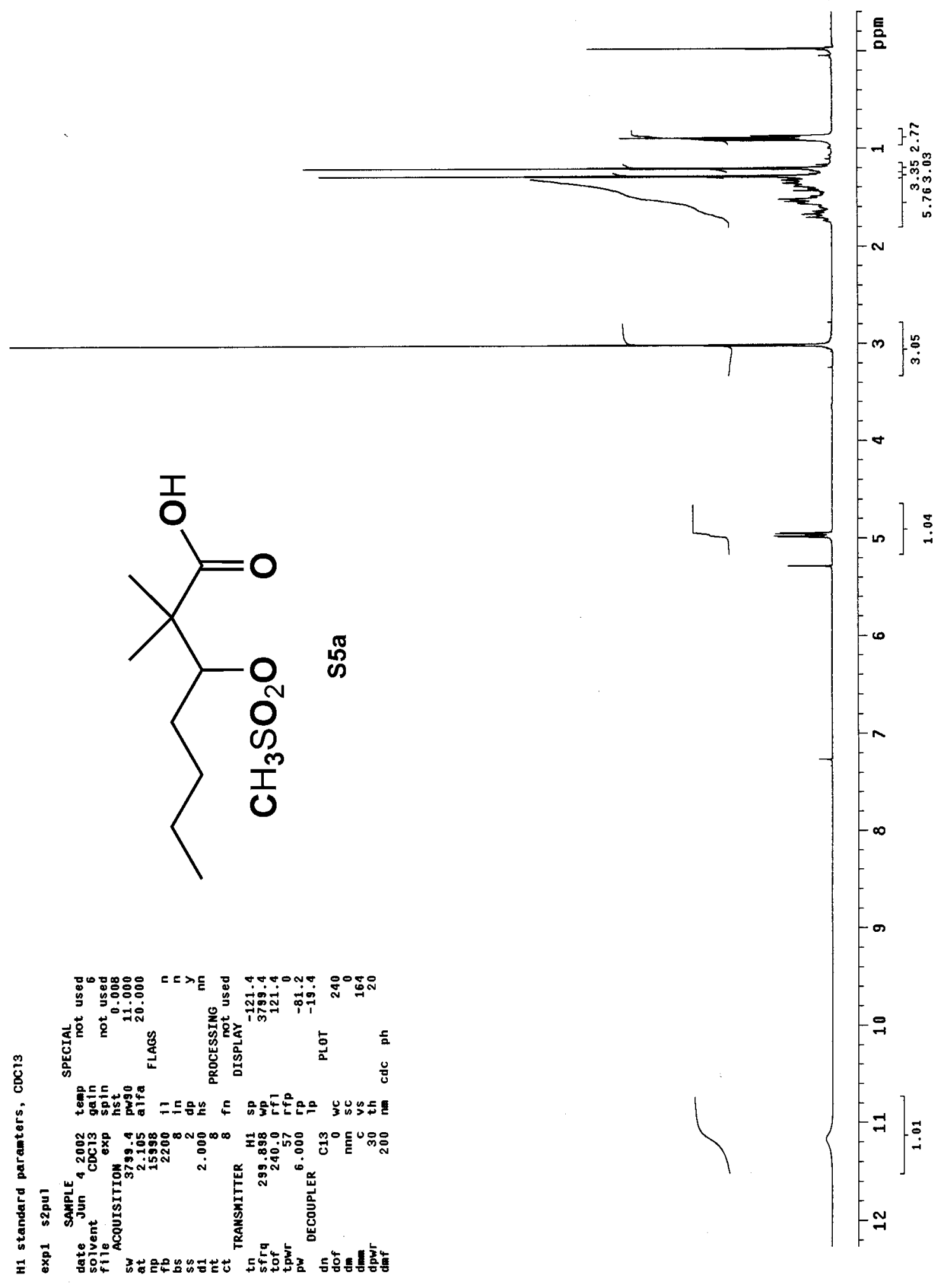




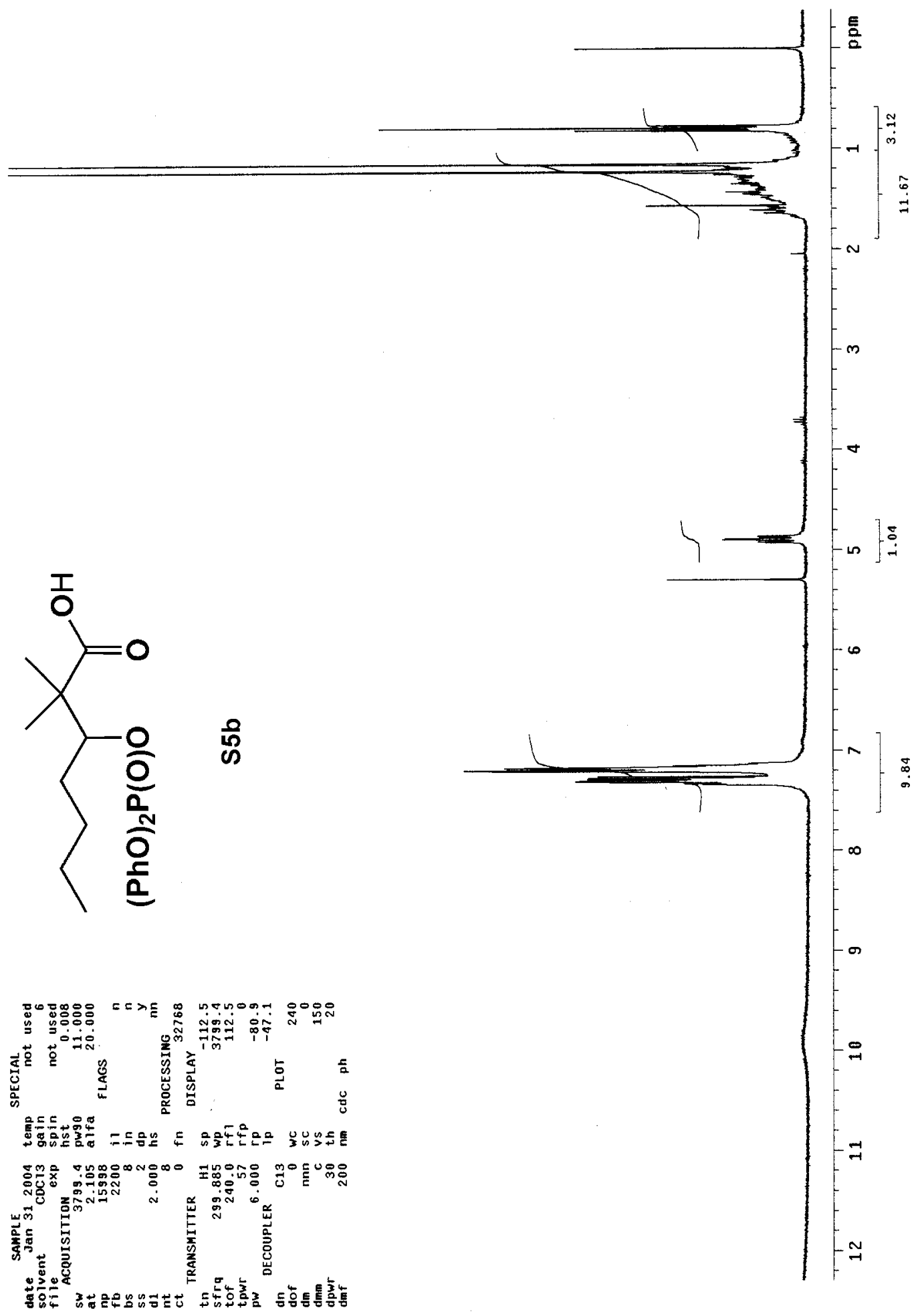




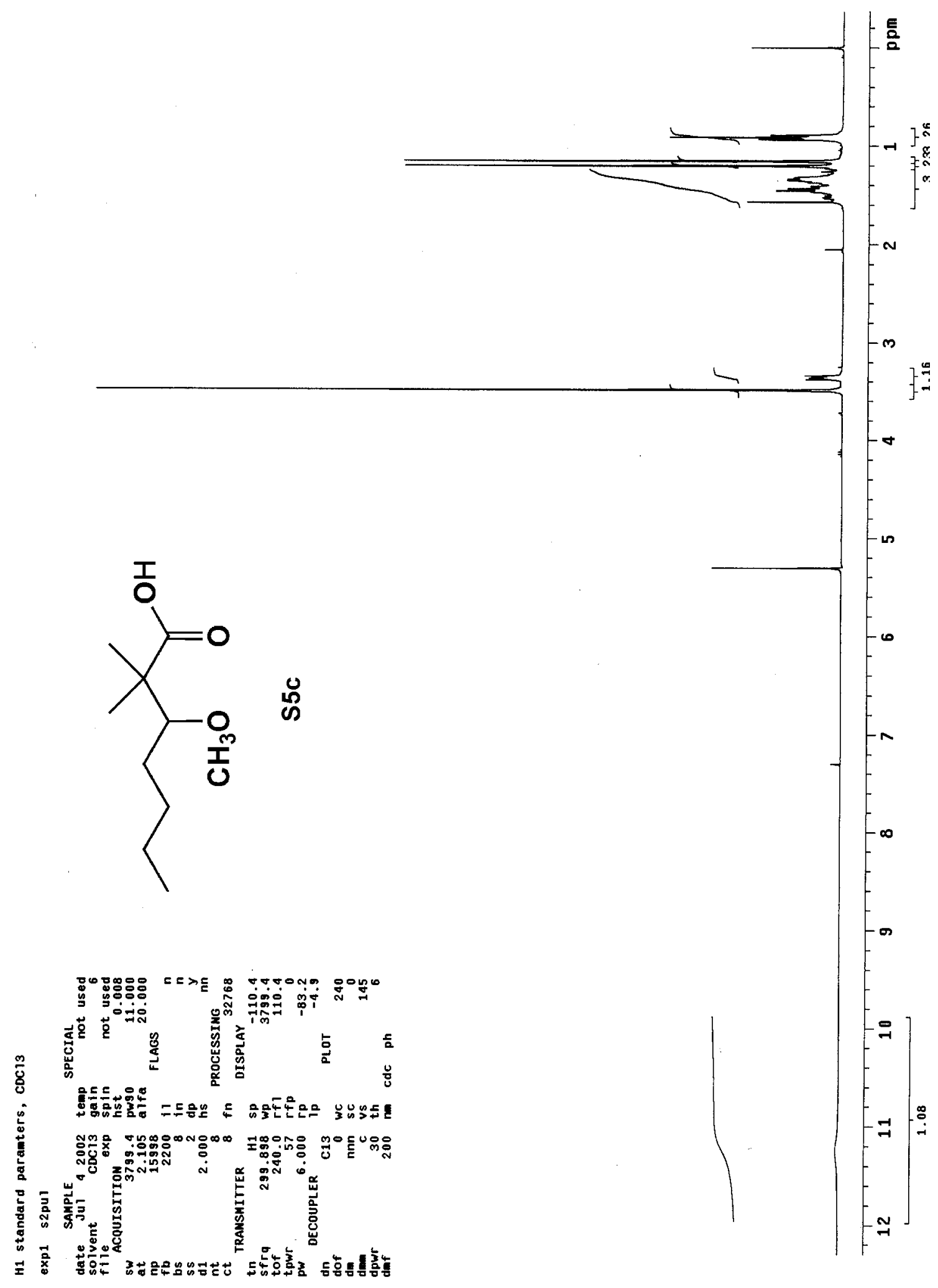




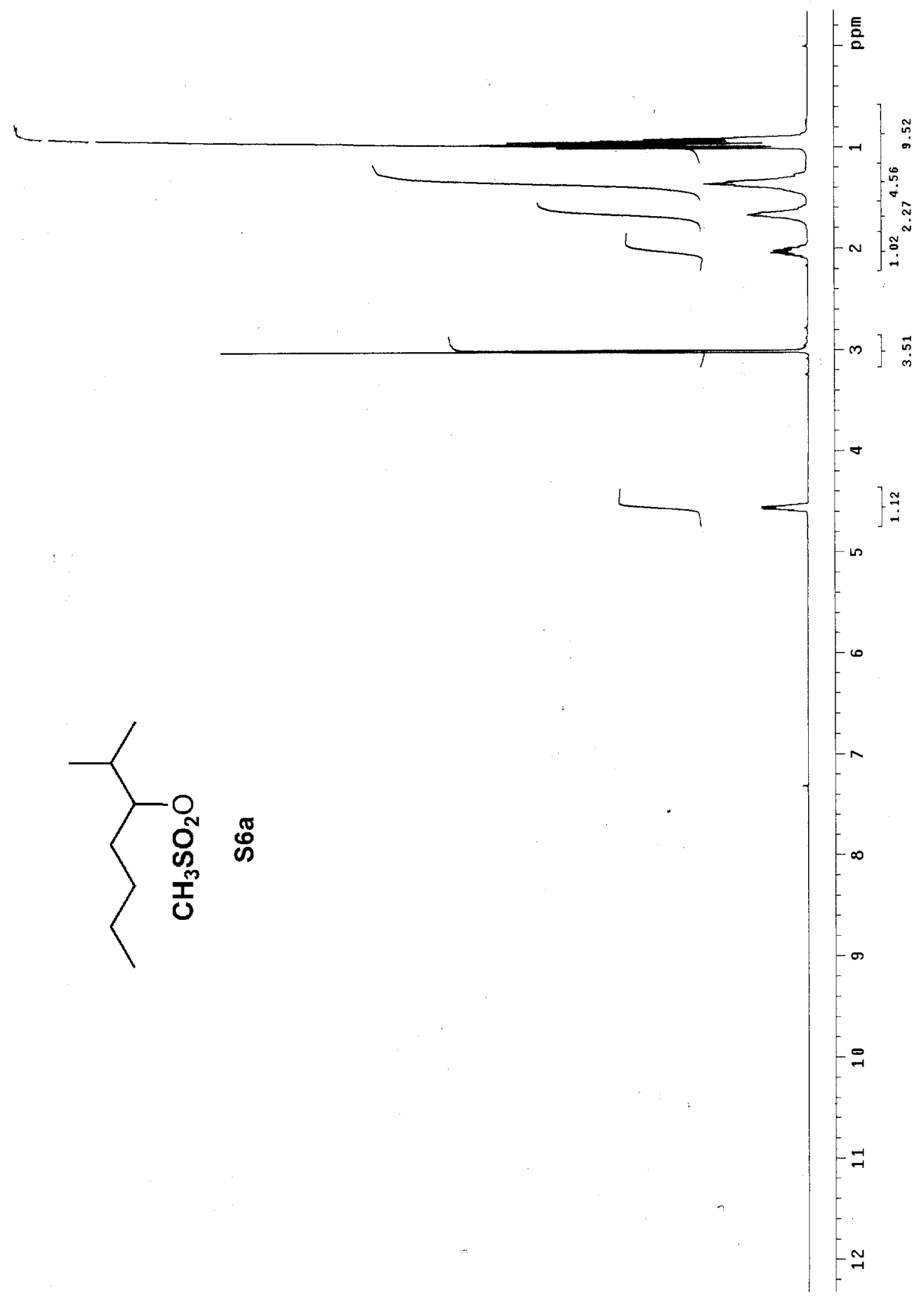




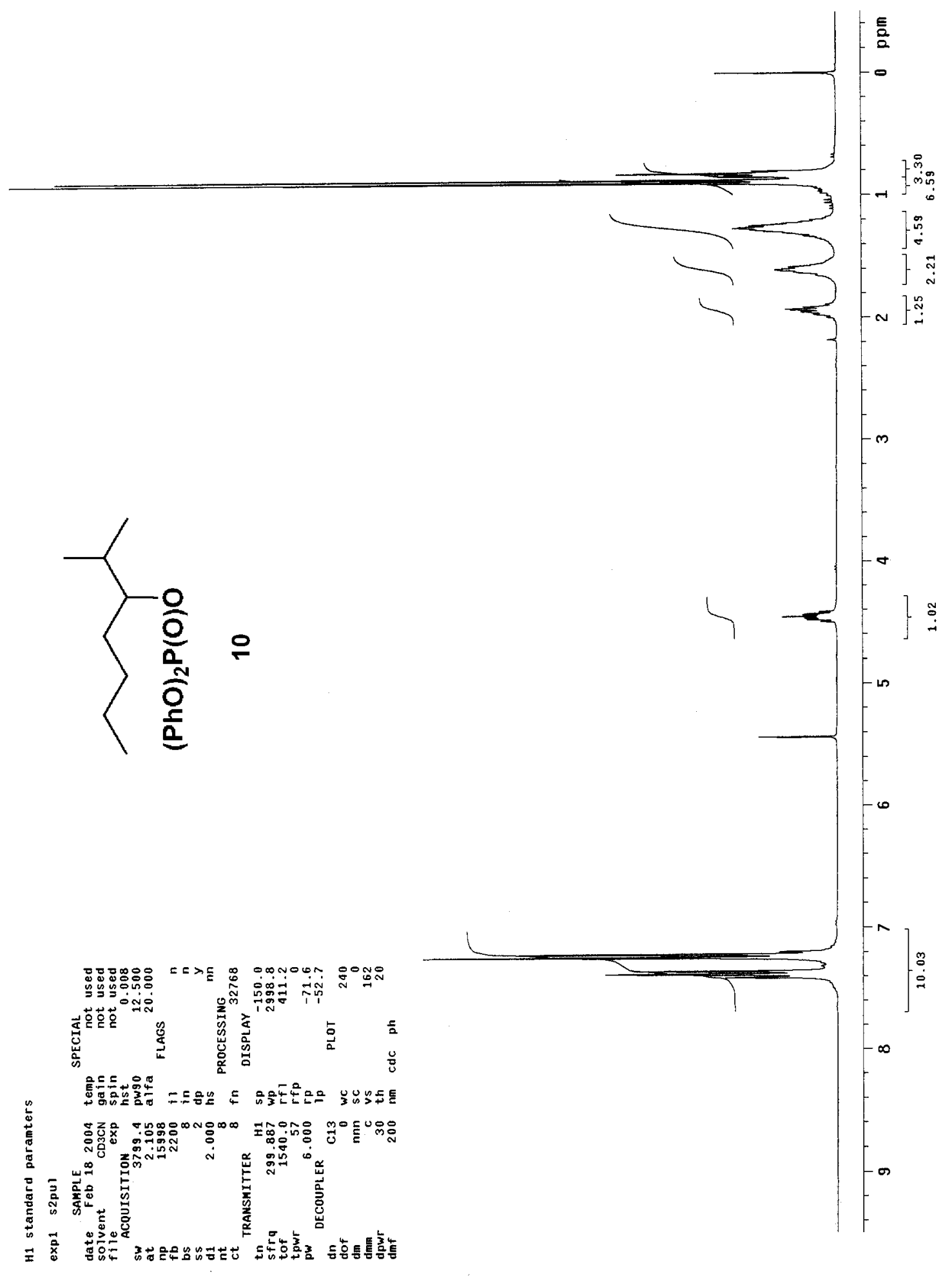




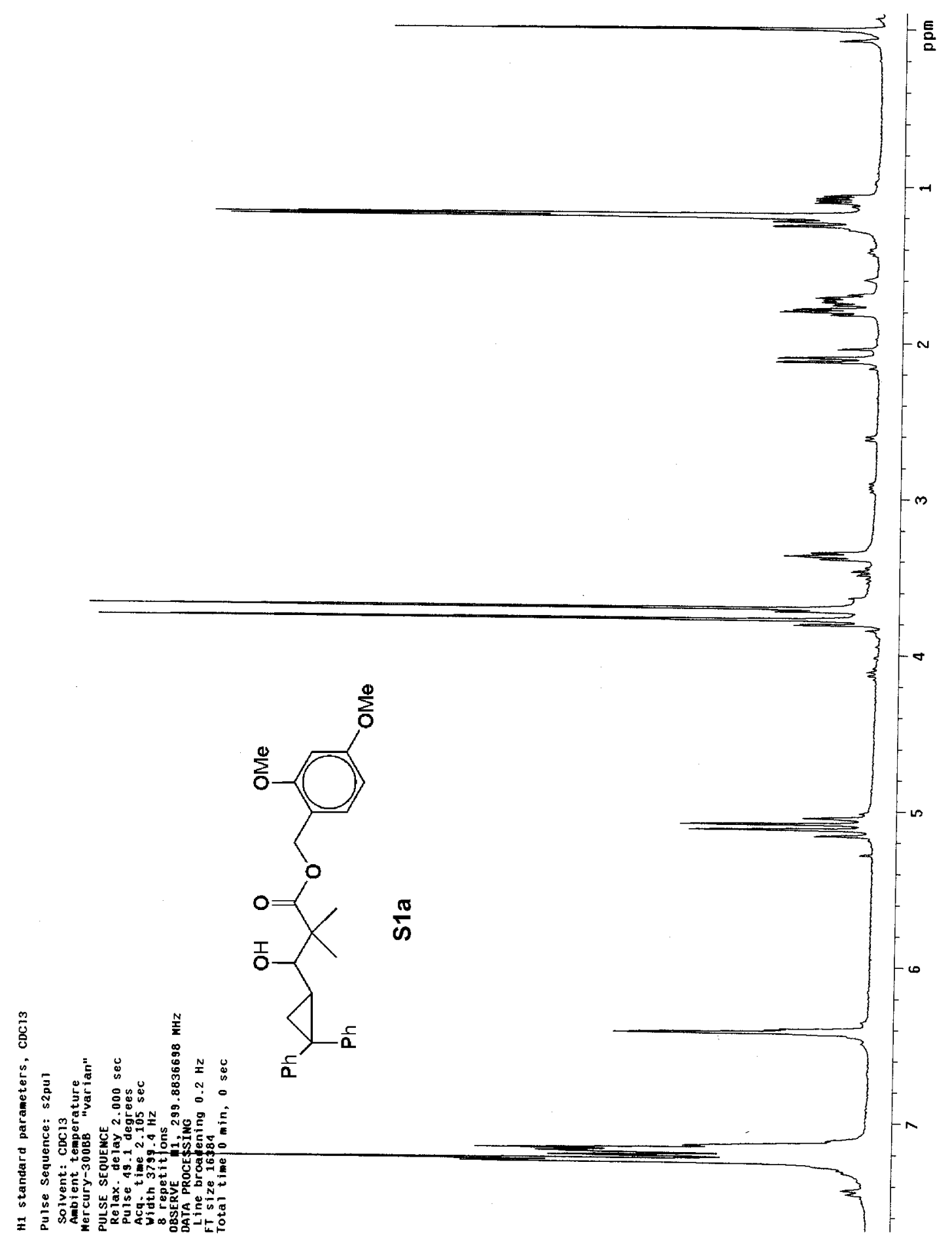




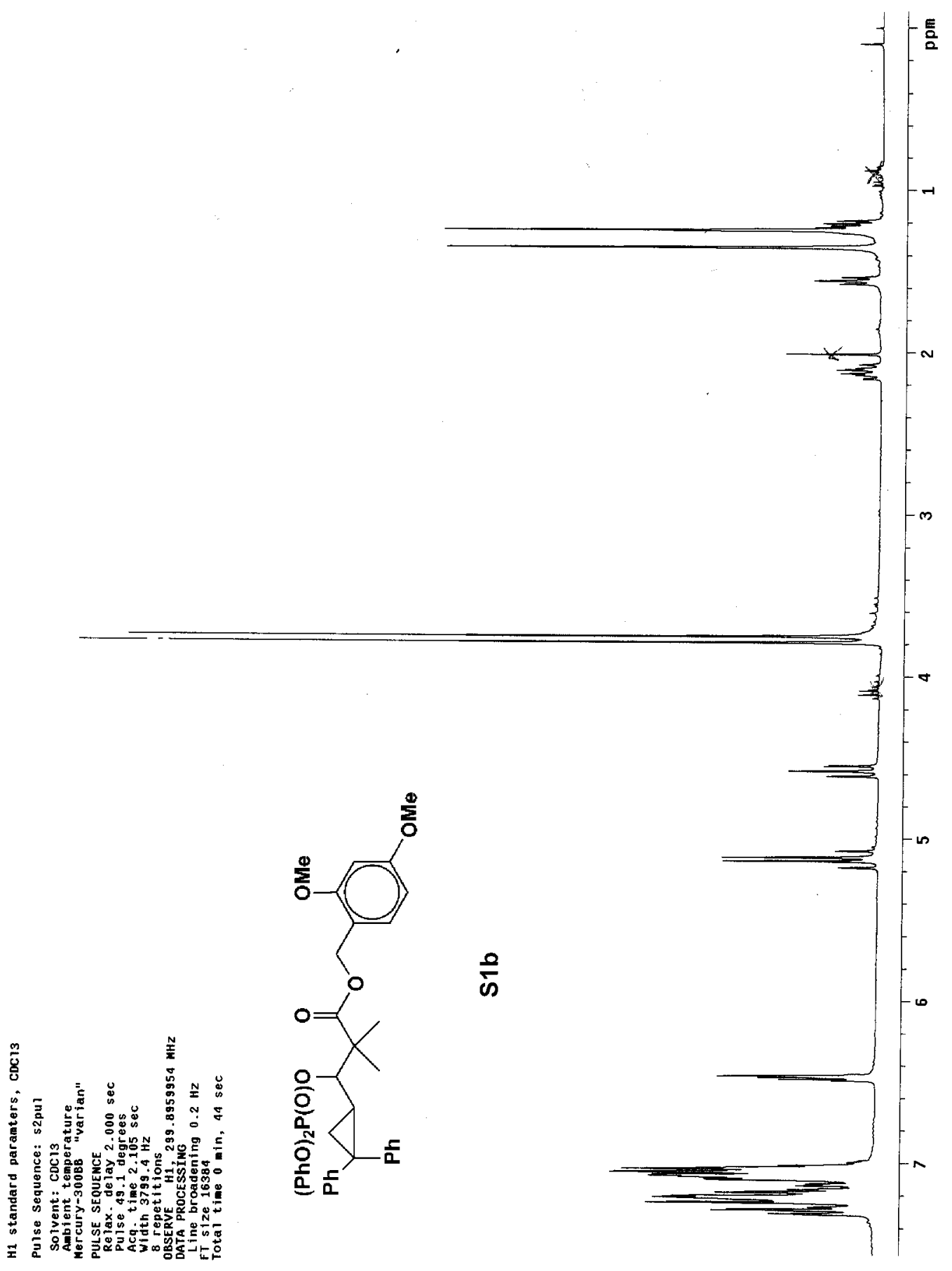




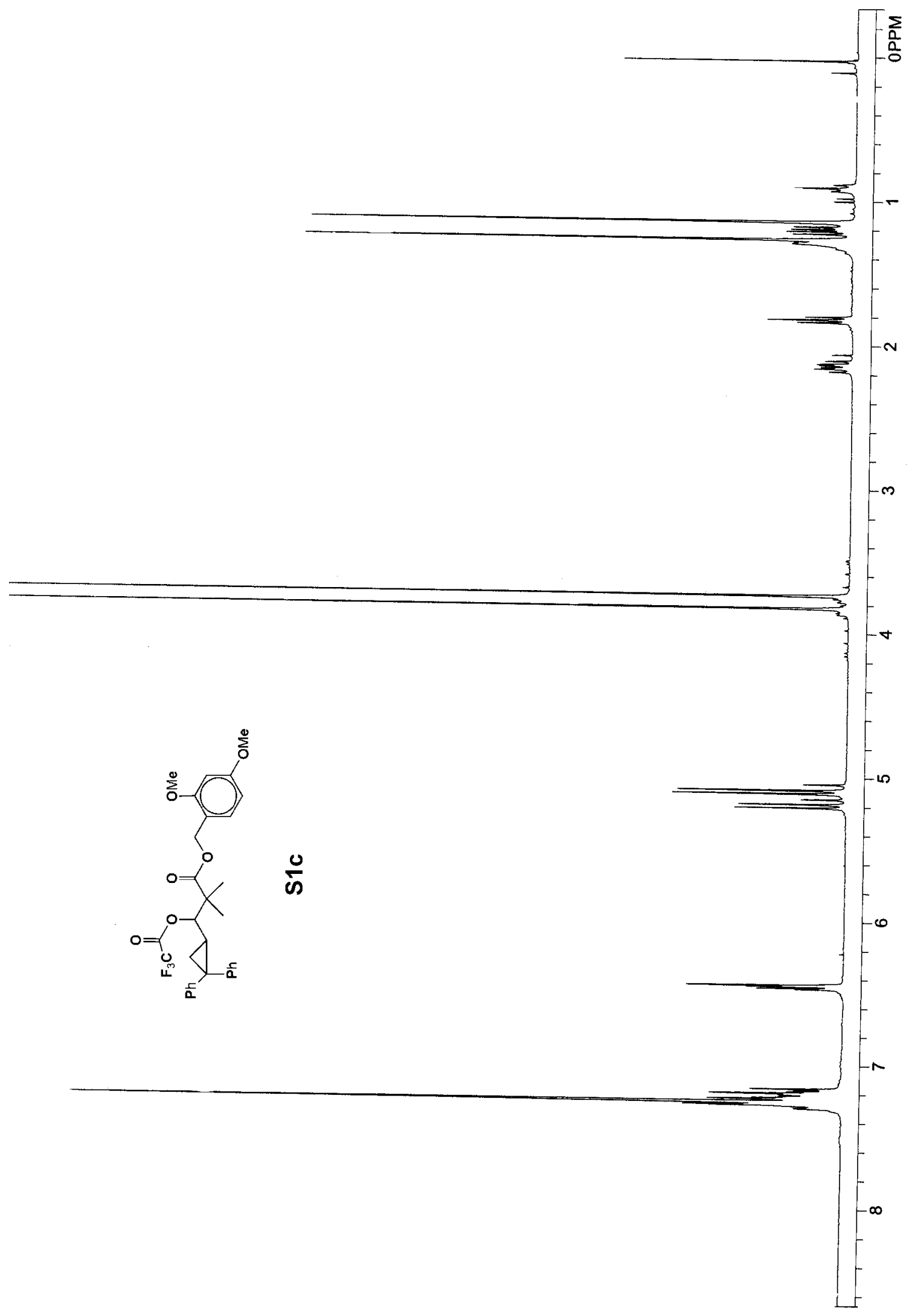




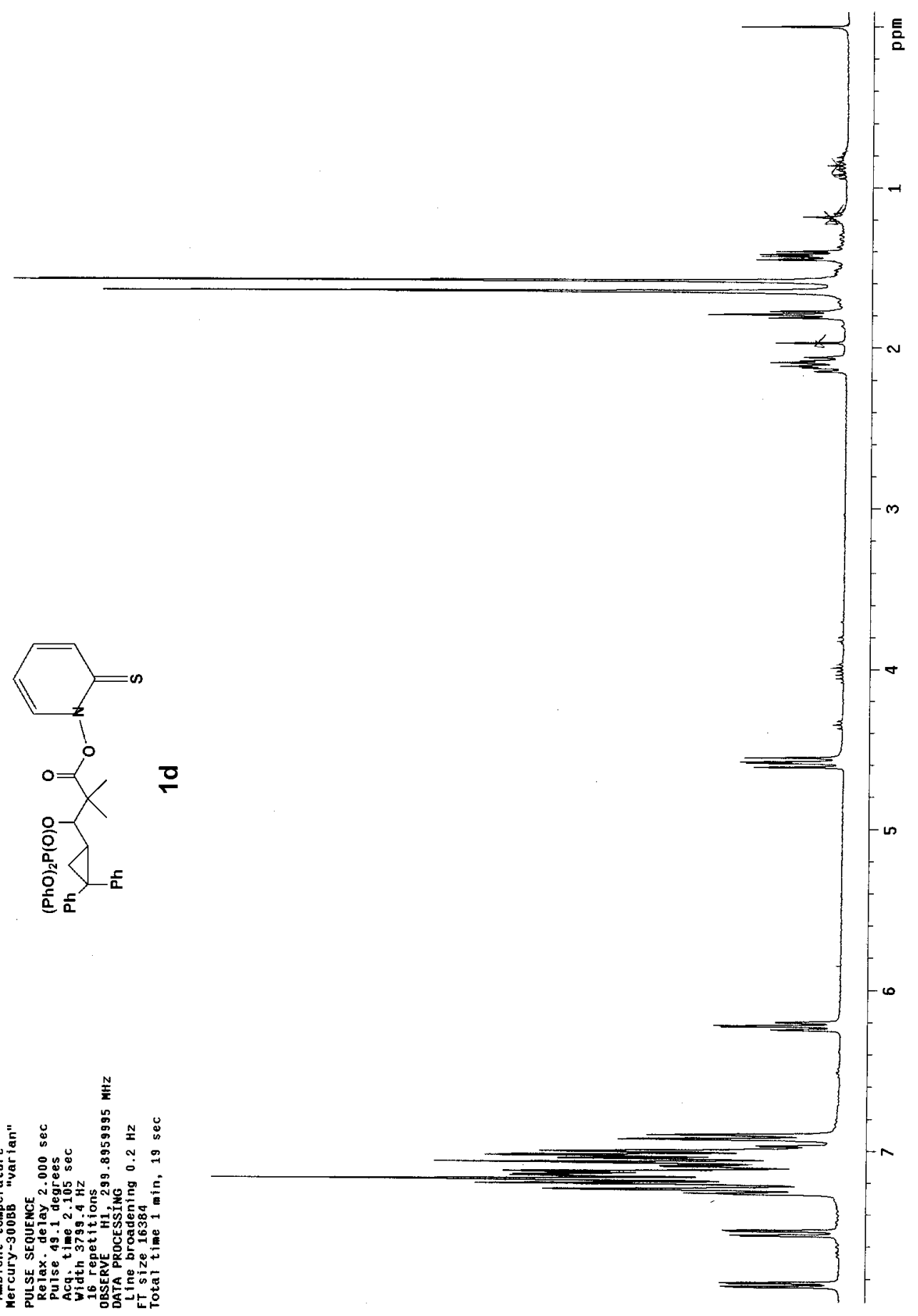




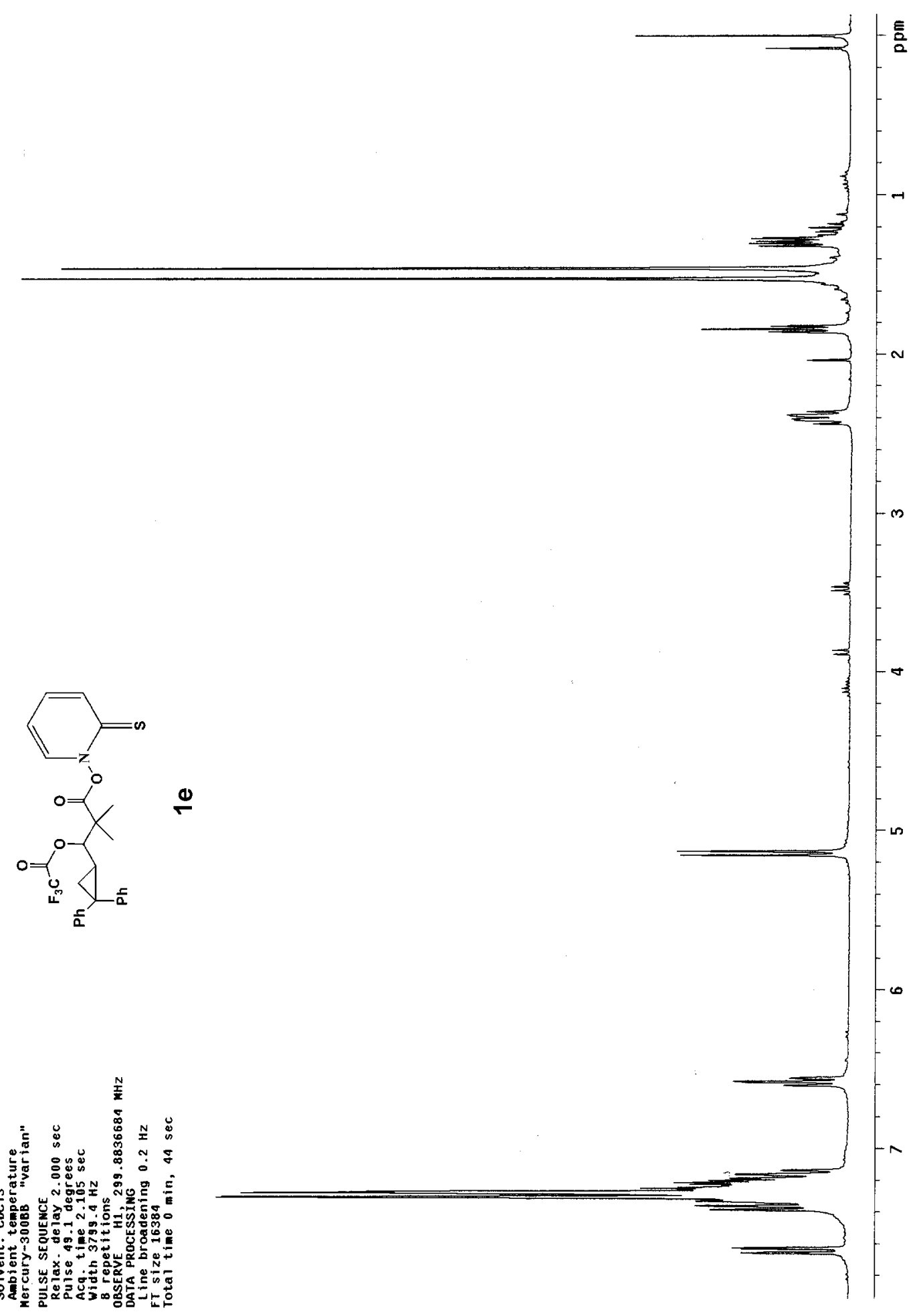

